\title{
Boundedness of Solutions in a Fully Parabolic Quasilinear Chemotaxis Model with Two Species and Two Chemicals
}

\author{
Aichao Liu and Binxiang Dai*
}

\begin{abstract}
This paper deals with a chemotaxis model with nonlinear signal production in a smoothly bounded domain. When there is no logistic growth source, the solutions of the system are globally bounded. This is also true if the logistic damping effect is strong enough. We extend recent research on single-species and one stimulus obtained by Tao et al. (2019, J. Math. Anal. Appl.) to two species chemotaxis system with two chemicals by creating an extra subtle inequality. We also partially extended some other related work.
\end{abstract}

\section{Introduction}

Chemotaxis refers to the guided migration of cells under the guidance of chemical gradients, which is crucial for a variety of biological processes. Chemotaxis has been confirmed in many processes: including patterning of the slime mold Dictyostelium, embryonic morphogenesis, wound healing, and tumor invasion (see 32 and therein). Continuous models of chemotaxis have been developed to describe many such systems.

The following classical Keller-Segel model [20] was originally used to describe the accumulation of Dictyostelium discotylum 21.

$$
\begin{cases}u_{t}=\Delta u-\chi \nabla \cdot(u \nabla v), & x \in \Omega, t>0, \\ \tau v_{t}=\Delta v-v+u, & x \in \Omega, t>0, \\ \frac{\partial u}{\partial \nu}=\frac{\partial v}{\partial \nu}=0, & x \in \partial \Omega, t>0 \\ (u, \tau v)(x, 0)=\left(u_{0}(x), \tau v_{0}(x)\right), & x \in \Omega,\end{cases}
$$

where $u$ represents the density of Dictyostelium discotylum, $v$ stands for the chemical concentration of an attractant or repellent. Here $\Omega \subset \mathbb{R}^{n}(n \geq 1)$ is a smoothly bounded domain and $\tau \geq 0$. The first equation of system 1.1) implies that the cell movement is directed toward (i.e., $\chi>0$ ) the increasing chemoattractant concentration or away from

Received June 2, 2021; Accepted October 17, 2021.

Communicated by Cheng-Hsiung Hsu.

2020 Mathematics Subject Classification. 35A01, 35K51, 35K55, 92C17.

Key words and phrases. boundedness, fully parabolic system, quasilinear, nonlinear signal production. This research is supported by the National Natural Science Foundation of China (No. 11871475).

*Corresponding author. 
(i.e., $\chi<0)$ the increasing chemorepellent concentration. The parameter $\chi$ measures the strength of the attraction or repulsion. The second equation of system (1.1) states that the chemoattractant or chemorepellent is produced by cells and undergoes decay. Model (1.1) is the basis of chemotactic models and has been studied deeply in the past four decades. To the best of our knowledge, the results seem quite complete (see, for example, $2,14,16,47]$ ). The important results are listed below: no blow-up in one dimensional [15, 31, 52] except for some extreme nonlinear diffusion models [3,6], critical mass blow-up in two dimensional 14, 16 and generic blow-up in higher dimensional [47.

Since the blow-up is an extreme case, a large amount of efforts were devoted to revising the model (1.1) such that the modified models allow global bounded solutions and thus produce patterns suitable for reality. Subsequently, various mechanisms such as adding logistic dampening $24,30,45]$, nonlinear variants with chemotactic sensitivity and diffusivity (see [38] and references therein) as well as the volume-filling effect [4, 33, 46], have been introduced into the system (1.1) to prevent the finite-time blow-up. Therefore, some scholars have studied the following chemotaxis model for two coupled equations

$$
\begin{cases}u_{t}=\nabla \cdot(D(u) \nabla u)-\nabla \cdot(S(u) \nabla v)+f(u), & x \in \Omega, t>0, \\ \tau v_{t}=\Delta v-v+g(u), & x \in \Omega, t>0, \\ \frac{\partial u}{\partial \nu}=\frac{\partial v}{\partial \nu}=0, & x \in \partial \Omega, t>0, \\ (u, \tau v)(x, 0)=\left(u_{0}(x), \tau v_{0}(x)\right), & x \in \Omega .\end{cases}
$$

Here $\tau \in\{0,1\}$, the positive function $D(u)$ and nonnegative function $S(u)$ stand for the diffusivity and chemotactic sensitivity of the cells respectively, the functions $f(u)$ and $g(u)$ indicate the growth of $u$ and production of $v$ respectively as well as the term $S(u) \nabla v$ refers to the cell movement towards the higher concentration gradient of the chemical signal. It is worth mentioning that Painter and Hillen [33] initially emphasized the importance of both nonlinear diffusion $D(u)$ and sensitivity $S(u)$. For chemotaxis models like 1.2, one of the most important problems is whether solutions remain bounded or blow up in finite/infinite time.

In the case of $g(u)=u$ and there is no source (i.e., $f \equiv 0$ ), when $\tau=1$, Horstmann and Winkler 17. proved that there exist radially symmetric solutions which blow up under the condition that $D(u)=1, S(u) \geq c_{0} u^{\alpha}$ with $c_{0}>0$ and $\alpha>\frac{2}{n}$ as well as $\Omega \subset \mathbb{R}^{n}(n \geq 2)$ is a ball. Subsequently, Winkler [46] showed that there exist solutions which blow up in either finite or infinite time if $\frac{S(u)}{D(u)}$ grows faster than $u^{\frac{2}{n}}$ as $u \rightarrow \infty$ and some further technical conditions are fulfilled. Cieślak and Stinner [7] showed that there exist solutions which blow up in finite time if $S(s)$ is non-decay for any $s>0$ and some extra conditions are satisfied; meanwhile there exist solutions which blow up in infinite time if both $D(s)$ and $S(s)$ decay (i.e., in the sense of $\lim _{s \rightarrow \infty} D(s)=0$ and $\lim _{s \rightarrow \infty} S(s)=0$ ) and are confined to 
some other conditions. In the same year, Tao and Winkler [38] showed that solutions are bounded under the conditions that $\frac{S(u)}{D(u)} \leq c u^{\alpha}$ with $\alpha<\frac{2}{n}$ as well as $\Omega$ is a convex domain. Subsequently, Ishida et al. 19 generalized the result in 38 to non-convex domain. When there exists logistic source, Zheng [60] showed all solutions are global and bounded under the conditions that $D(u)=(1+u)^{-\alpha}, S(u)=u(1+u)^{\beta-1}$ and $f(u)=r-\mu u^{k}$ as well as $0<\alpha+\beta<\max \left\{k-1+\alpha, \frac{2}{n}\right\}$ or $\beta=k-1$ and $\mu$ is large enough. In addition, there are some studies on $\tau=0$ 51,59. In the case of general $g(u)$ and there is no source, Liu and Tao 25] obtained the global boundedness of system 1.2 with $\tau=1$ under the conditions that $D(u) \equiv 1, S(u) \equiv u$ and $g(u)=u^{\gamma}$ with $0<\gamma<\frac{2}{n}$. Subsequently, when $\tau=0$ and $\Omega \subset \mathbb{R}^{n}$ is a ball as well as $D(u) \equiv 1$ and $S(u) \equiv u$, Winkler [49 showed the solution is global and bounded under the conditions that $g(u) \leq K u^{\gamma}$ with $\gamma<\frac{2}{n}$ and $K>0$; meanwhile the solution blows up in finite time under the conditions that $g(u) \geq k u^{\gamma}$ with $\gamma>\frac{2}{n}$ and $k>0$. When there is logistic kinetics, the case with $\tau=0$ and $D(u)=1$ has been investigated $[10,18,62$. However, there is little literature on system 1.2 with $\tau=1$. On $\Omega \subseteq \mathbb{R}^{2}$, for functions $(D, S, g) \in\left(C^{2}([0, \infty))\right)^{3}$ fulfilling $D(s) \geq c_{0} s^{m-1}, S(s) \leq \chi_{0} s^{\alpha}, g(s) \leq \kappa_{0} s^{\beta}$ and $f(s)=r s-\mu s^{2}$ with $c_{0}>0, \chi_{0}>0$, $\kappa_{0}>0, m \in \mathbb{R}, \alpha>0, \beta \in(0,1], r \geq 0$ and $\mu>0$, Zheng et al. [63] obtained the global existence of bounded solutions under the conditions that $m>2 \alpha, \beta \in(0,1]$. Recently, on $\Omega \subseteq \mathbb{R}^{n}(n \geq 2)$, for functions $(D, S) \in\left(C^{2}([0, \infty))\right)^{2}, f \in C^{0}([0, \infty))$ and $g \in C^{1}([0, \infty))$ satisfying $d_{0}(1+s)^{-\alpha} \leq D(s) \leq d_{1}(1+s)^{-\alpha_{1}}, 0 \leq S(s) \leq s_{1} s(1+s)^{\beta-1}, f(s) \leq r s-\mu s^{k}$ and $0 \leq g(s) \leq g_{1} s^{\gamma}$ with $d_{0}>0, d_{1}>0, s_{1}>0, \alpha \in \mathbb{R}, \alpha_{1} \in \mathbb{R}, \beta \in \mathbb{R}, r \in \mathbb{R}, \mu>0$, $g_{1}>0, \gamma>0$ and $k>1$, Tao et al. 37] obtained the global existence of bounded solutions under the conditions that $f \equiv 0, \gamma \in(0,1], \alpha+\beta+\gamma<1+\frac{2}{n}$ or $f \not \equiv 0, \beta+\gamma<k$ or $f \not \equiv 0, \beta+\gamma=k, \mu \geq \mu_{0}$ for some $\mu_{0}>0$. In addition, there are some papers on the asymptotic behavior, to name a few, see [5, 8, 26, 54].

In nature, populations always interact with each other. Studies have confirmed that interactions of several populations via chemotactic mechanisms play an important role in various biological processes 13, 34]. So far, existing literature is scarce and mainly focuses on the case where there are two species and the two species produce the same signal $1,29,36,40,57$. In order to understand the chemotactic interaction in presence of several chemicals, Tao and Winkler [39] considered the following model involving two species and two signals,

$$
\begin{cases}u_{t}=\Delta u-\chi \nabla \cdot(u \nabla v), \quad \tau v_{t}=\triangle v-v+\omega, & (x, t) \in \Omega \times(0, \infty), \\ \omega_{t}=\triangle \omega-\xi \nabla \cdot(\omega \nabla z), \quad \tau z_{t}=\Delta z-z+u, & (x, t) \in \Omega \times(0, \infty), \\ \frac{\partial u}{\partial \nu}=\frac{\partial v}{\partial \nu}=\frac{\partial \omega}{\partial \nu}=\frac{\partial z}{\partial \nu}=0, & (x, t) \in \partial \Omega \times(0, \infty), \\ (u, \tau v, \omega, \tau z)(x, 0)=\left(u_{0}(x), \tau v_{0}(x), \omega_{0}(x), \tau z_{0}(x)\right), & x \in \Omega .\end{cases}
$$


Here $\tau \in\{0,1\}, u(x, t)$ and $\omega(x, t)$ denote the densities of the two species and the chemicals produced are $z(x, t)$ and $v(x, t)$ respectively. That is, besides diffusions of the two species themselves, one species produces a chemical signal to affect the motion of the other. The signs of $\chi \in \mathbb{R}$ and $\xi \in \mathbb{R}$ determine the types of interactions, attraction or repulsion. Model (1.3) can describe the chemotaxis driven cell sorting process [32, the spatio-temporal evolution of two populations whose individuals move according to random diffusion, and chemotactically directed motion leading to an interaction in a circular manner.

When $\tau=0$, Tao and Winkler 39 showed that system 1.3 possesses a unique bounded classical solution whenever $\chi=-1$ and $n \leq 3$ or $\xi=-1$ and $n \leq 3$; and under the cases of $\chi=\xi=1$ and $n=2$, the similar result holds if $\max \left\{\left\|u_{0}\right\|_{L^{1}(\Omega)},\left\|\omega_{0}\right\|_{L^{1}(\Omega)}\right\}<$ $\frac{4}{C_{G N}}$; in addition, blow-up may occur if $n=2$ and $\min \left\{\left\|u_{0}\right\|_{L^{1}(\Omega)},\left\|\omega_{0}\right\|_{L^{1}(\Omega)}\right\}>4 \pi$. Subsequently, some scholars obtained the boundedness criteria and blow-up criteria of solutions in lower dimensional space 23, 55, 61. However, in a varying biological environment, according to the classical Lotka-Volterra kinetics [28], cells may proliferate and compete for resources and space in order to survive. Therefore, proliferation and competition ingredients are incorporated into system (1.3). At that moment, most of works in this direction mainly investigated the boundedness, stability, and convergence rate of solutions when the logistic damping effect is strong enough (for example, see, $35,41,43,56,58,64$ ).

In a changing biological environment, the movement of cells involved in two species and two chemicals should be described by nonlinear functions similar to the system (1.2). As far as we know, for model (1.3), there is no similar work as system 1.2 due to technical difficulties. This inspires us to consider the following system

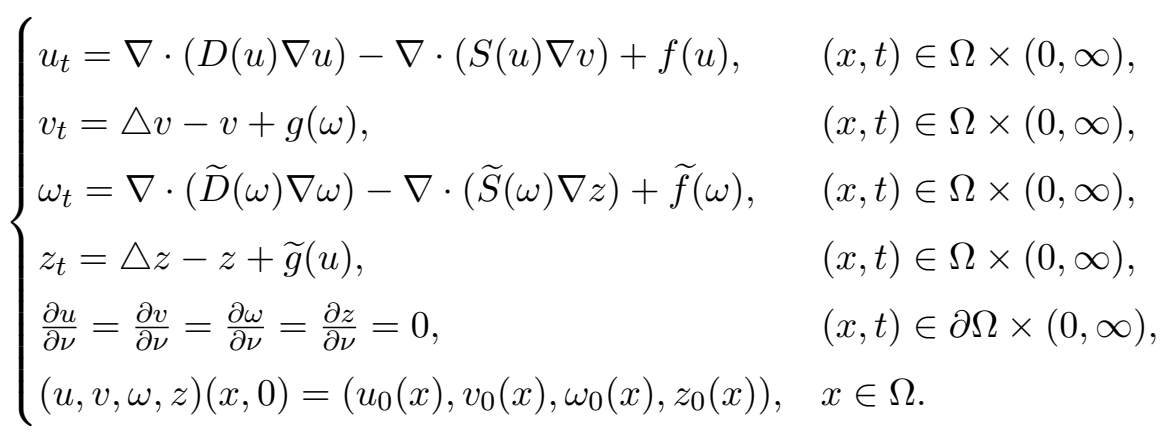

For the convenience of research, throughout this paper, we assume that the nonnegative initial data $\left(u_{0}, v_{0}, \omega_{0}, z_{0}\right)$ satisfy

$$
\left(u_{0}, v_{0}, \omega_{0}, z_{0}\right) \in C^{0}(\bar{\Omega}) \times C^{1}(\bar{\Omega}) \times C^{0}(\bar{\Omega}) \times C^{1}(\bar{\Omega}), \quad u_{0} \not \equiv 0, \quad \omega_{0} \not \equiv 0 .
$$

The functions $D, S, \widetilde{D}, \widetilde{S} \in C^{2}([0, \infty))$ satisfy $S(0)=0$ and $\widetilde{S}(0)=0$ as well as

$$
\begin{array}{ll}
d_{0}(1+u)^{-\alpha} \leq D(u) \leq d_{1}(1+u)^{-\alpha_{1}}, & 0 \leq S(u) \leq s_{1} u(1+u)^{\beta-1}, \\
\widetilde{d}_{0}(1+\omega)^{-\widetilde{\alpha}} \leq \widetilde{D}(\omega) \leq \widetilde{d}_{1}(1+\omega)^{-\widetilde{\alpha}_{1}}, & 0 \leq \widetilde{S}(\omega) \leq \widetilde{s}_{1} \omega(1+\omega)^{\widetilde{\beta}-1}
\end{array}
$$


for all $u, \omega \geq 0$ with some $d_{0}, d_{1}, s_{1}, \widetilde{d}_{0}, \widetilde{d}_{1}, \widetilde{s}_{1}>0$ and $\alpha, \alpha_{1}, \beta, \widetilde{\alpha}, \widetilde{\alpha}_{1}, \widetilde{\beta} \in \mathbb{R}$. Moreover, we assume that $f, \tilde{f} \in C^{2}([0, \infty))$ with $f(0), \tilde{f}(0) \geq 0$ and $g, \widetilde{g} \in C^{2}([0, \infty))$ fulfill

$$
\begin{array}{lll}
f(u) \leq r u-\mu u^{k}, & 0 \leq \widetilde{g}(u) \leq \widetilde{\lambda}_{1} u^{\widetilde{\gamma}} & \text { for all } u \geq 0, \\
\widetilde{f}(\omega) \leq \widetilde{r} \omega-\widetilde{\mu} \omega^{\widetilde{k}}, & 0 \leq g(\omega) \leq \lambda_{1} \omega^{\gamma} & \text { for all } \omega \geq 0,
\end{array}
$$

where $r, \widetilde{r} \in \mathbb{R}, \mu, \lambda_{1}, \gamma, \widetilde{\mu}, \widetilde{\lambda}_{1}, \widetilde{\gamma}>0$ and $k, \widetilde{k}>1$.

The goal of this paper is to establish global existence and boundedness of classical solution of system (1.4) under the assumptions (1.5)-(1.7). Precisely, we obtain the following main results.

Theorem 1.1. Let $\Omega \subset \mathbb{R}^{n}(n \geq 2)$ be a bounded domain with smooth boundary, $f=\widetilde{f} \equiv 0$ as well as nonnegative initial data $\left(u_{0}, v_{0}, \omega_{0}, z_{0}\right)$ satisfy $(1.5)$. Suppose $D, S, \widetilde{D}, \widetilde{S}$ and $g, \widetilde{g}$ fulfill (1.6) and (1.7) respectively. If $\gamma, \widetilde{\gamma} \in(0,1]$ and $\alpha_{1}=\widetilde{\alpha}_{1}$ as well as

$$
\alpha=\widetilde{\alpha}<\frac{2}{n} \quad \text { and } \quad \alpha+\max \{\beta+\gamma, \widetilde{\beta}+\widetilde{\gamma}\}<1+\frac{2}{n},
$$

then problem (1.4) admits a nonnegative classical solution $(u, v, \omega, z)$ which is globally bounded.

Theorem 1.1 tells us that nonlinear variants involved in diffusivity, chemotactic sensitivity and signal production are beneficial to the global existence of the solution when there is no growth source. Theorem 1.1 also extends the study in [61] to the fully parabolic quasilinear chemotaxis model.

Theorem 1.2. Let $n \geq 2$ and nonnegative initial data $\left(u_{0}, v_{0}, \omega_{0}, z_{0}\right)$ satisfy (1.5). Suppose $D, S, \widetilde{D}, \widetilde{S}$ and $f, \widetilde{f}, g, \widetilde{g}$ fulfill (1.6) and (1.7) respectively, if $k=\widetilde{k}, \beta=\widetilde{\beta}$ and one of the following is true

(1) $\max \{\gamma, \widetilde{\gamma}\}<k-\beta$;

(2) $\widetilde{\gamma}<\gamma=k-\beta, \mu>2^{k+1} s_{1}, \widetilde{\mu}>\widetilde{c}_{p} ;$

(3) $\gamma<\widetilde{\gamma}=k-\beta, \mu>c_{p}, \widetilde{\mu}>2^{k+1} \widetilde{s}_{1}$;

(4) $\gamma=\widetilde{\gamma}=k-\beta, \mu>\max \left\{2^{k+1} s_{1}, c_{p}\right\}, \widetilde{\mu}>\max \left\{2^{k+1} \widetilde{s}_{1}, \widetilde{c}_{p}\right\}$,

then problem (1.4) possesses a nonnegative classical solution $(u, v, \omega, z)$ which is globally bounded. Here $c_{p}$ and $\widetilde{c}_{p}$ are defined in (3.41) and (3.39) respectively.

Theorem 1.2 shows that strong logistic damping effect is conducive to the global existence of solutions. Theorem 1.2 coincides with that in 37 when two species and signals are exactly the same in system (1.4).

Before we prove the main results in the third Section, we first show the local existence of a classical solution to (1.4) and provide some preliminary results. The paper ends with a brief summary and discussion. 


\section{Local existence and preliminaries}

The proof of the local existence of solutions in time is similar to those in [24,37, which is achieved by employing a fixed point theorem. Though we will not give the detail, for readers' convenience, we cite some used knowledge related to the Neumann heat semigroup. We state it here (for instance, see [17,44]). The operator $A=:-\triangle+1$ is sectorial in $L^{p}(\Omega)$ and therefore admits closed fractional powers $A^{\theta}, \theta \in(0,1)$ with dense domain $D\left(A^{\theta}\right)$. Two basic and useful properties of $A^{\theta}$ are listed below.

(P1) If $m \in\{0,1\}, p \in[1, \infty]$ and $q \in(1, \infty)$ with $m-\frac{n}{p}<2 \theta-\frac{n}{q}$, then there exists some positive constant $C$ such that

$$
\|\phi\|_{W^{m, p}(\Omega)} \leq C\left\|A^{\theta} \phi\right\|_{L^{q}(\Omega)} \quad \text { for all } \phi \in D\left(A^{\theta}\right) .
$$

(P2) For $p<\infty$, the Neumann heat semigroup $\left(e^{t \triangle}\right)_{t \geq 0}$ maps $L^{p}(\Omega)$ into $D\left(A^{\theta}\right)$ in any of the space $L^{q}(\Omega)$ for $q \geq p$, and there exist $C>0$ and $\mu>0$ such that

$$
\left\|A^{\theta} e^{-A t} \phi\right\|_{L^{q}(\Omega)} \leq C t^{-\theta-\frac{n}{2}\left(\frac{1}{p}-\frac{1}{q}\right)} e^{-\mu t}\|\phi\|_{L^{p}(\Omega)} \quad \text { for all } \phi \in L^{p}(\Omega) .
$$

Proposition 2.1. Let $n \geq 1$ and nonnegative initial data $\left(u_{0}, v_{0}, \omega_{0}, z_{0}\right)$ satisfy (1.5). Suppose $D, S, \widetilde{D}, \widetilde{S}$ and $f, \widetilde{f}, g, \widetilde{g}$ fulfill (1.6) and (1.7) respectively. Then system 1.4 admits a nonnegative local-in-time classical solution $(u, v, \omega, z) \in\left(C^{0}\left(\bar{\Omega} \times\left[0, T_{\text {max }}\right)\right) \cap\right.$ $\left.C^{2,1}\left(\bar{\Omega} \times\left(0, T_{\max }\right)\right)\right)^{4}$. Here $T_{\max }$ denotes the maximum existence time. Moreover, if $T_{\max }<\infty$, then

$$
\limsup _{t \rightarrow T_{\max }-}\left(\|u\|_{L^{\infty}(\Omega)}+\|v\|_{L^{\infty}(\Omega)}+\|\omega\|_{L^{\infty}(\Omega)}+\|z\|_{L^{\infty}(\Omega)}\right)=\infty
$$

The following result shows some fundamental properties of solution $(u, v, \omega, z)$ to problem (1.4) without logistic source.

Lemma 2.2. Let $n \geq 1, f=\widetilde{f} \equiv 0$ and nonnegative initial data $\left(u_{0}, v_{0}, \omega_{0}, z_{0}\right)$ satisfy (1.5). Suppose $D, S, \widetilde{D}, \widetilde{S}$ and $g, \widetilde{g}$ fulfill (1.6) and (1.7) respectively, then the mass of $u$ and $\omega$ is conserved in the sense that

$$
\|u\|_{L^{1}(\Omega)}=\left\|u_{0}\right\|_{L^{1}(\Omega)}, \quad\|\omega\|_{L^{1}(\Omega)}=\left\|\omega_{0}\right\|_{L^{1}(\Omega)} \quad \text { for all } t \in\left(0, T_{\max }\right) .
$$

Moreover, if $\gamma \in(0,1]$ and $\widetilde{\gamma} \in(0,1]$, then for any $s \in\left[1, \frac{n}{(n \gamma-1)_{+}}\right) \cap\left[1, \frac{n}{(n \tilde{\gamma}-1)_{+}}\right)$, there exist positive constants $C_{1}=C_{1}(s, \gamma)$ and $C_{2}=C_{2}(s, \widetilde{\gamma})$ such that

$$
\|v\|_{W^{1, s}(\Omega)} \leq C_{1} \quad \text { and } \quad\|z\|_{W^{1, s}(\Omega)} \leq C_{2} \quad \text { for all } t \in\left(0, T_{\max }\right) .
$$

Here $\varsigma_{+}=\max \{\varsigma, 0\}$. 
Proof. Integrating the first and third equations in $(1.4)$ over $\Omega$, we deduce $\frac{d}{d t} \int_{\Omega} u=0$ and $\frac{d}{d t} \int_{\Omega} \omega=0$ which imply $(2.3)$. The assertion 2.4 can be obtained on the basis of a method of Neumann semigroup estimates 22, 37,53, so we will not give the detail.

Next, the following Lemma plays an important role in removing convexity of domains.

Lemma 2.3. 27. Let $n \geq 1$ and $\Phi \in C^{2}(\bar{\Omega})$, if $\left.\frac{\partial \Phi}{\partial \nu}\right|_{\partial \Omega}=0$, then

$$
\frac{\partial|\nabla \Phi|^{2}}{\partial \nu} \leq 2 \kappa_{\Omega}|\nabla \Phi|^{2} \quad \text { on } \partial \Omega
$$

where $\kappa_{\Omega}>0$ is an upper bound for the curvatures of $\partial \Omega$.

Then, we follow the ideas of [38, Lemma 3.1] and [17, Lemma 4.2] to establish the following result which plays an important role in proving Proposition 3.1 .

Proposition 2.4. Assume $\rho \in(0,1)$ and $\varrho \in(0,1)$ as well as $\rho+\varrho<1$, then for any $\eta_{1}>0$ and $\eta_{2}>0$, there exists some positive constant $C=C\left(\eta_{1}, \eta_{2}, \rho, \varrho\right)$ such that

$$
a^{\rho} b^{\varrho} \leq \eta_{1} a+\eta_{2} b+C
$$

where $a \geq 0$ and $b \geq 0$.

Proof. Firstly, for any $\eta_{1}>0$ and $\eta>0$, applying Young's inequality to the terms $a^{\rho} b^{\varrho}$ and $b^{\frac{\varrho}{1-\rho}}$ respectively produces

$$
a^{\rho} b^{\varrho} \leq \eta_{1} a+(1-\rho)\left(\frac{\eta_{1}}{\rho}\right)^{-\frac{\rho}{1-\rho}} \cdot b^{\frac{\varrho}{1-\rho}} \quad \text { and } \quad b^{\frac{\varrho}{1-\rho}} \leq \frac{\varrho \eta}{1-\rho} b+\frac{1-(\rho+\varrho)}{1-\rho} \eta^{-\frac{\varrho}{1-(\rho+\varrho)}}
$$

Next, we insert the second inequality into the first inequality to get

$$
a^{\rho} b^{\varrho} \leq \eta_{1} a+\left(\frac{\eta_{1}}{\rho}\right)^{-\frac{\rho}{1-\rho}} \varrho \eta b+\left(\frac{\eta_{1}}{\rho}\right)^{-\frac{\rho}{1-\rho}}[1-(\rho+\varrho)] \eta^{-\frac{\varrho}{1-(\rho+\varrho)}} .
$$

Finally, substituting $\eta=\frac{\eta_{2}}{\varrho}\left(\frac{\eta_{1}}{\rho}\right)^{\frac{\rho}{1-\rho}}$ into the above inequality yields

$$
a^{\rho} b^{\varrho} \leq \eta_{1} a+\eta_{2} b+\left(\frac{\eta_{1}}{\rho}\right)^{-\frac{\rho}{1-(\rho+\varrho)}} \cdot\left(\frac{\eta_{2}}{\varrho}\right)^{-\frac{\varrho}{1-(\rho+\varrho)}} \cdot[1-(\rho+\varrho)]=\eta_{1} a+\eta_{2} b+C,
$$

where $C=\left(\frac{\eta_{1}}{\rho}\right)^{-\frac{\rho}{1-(\rho+\varrho)}} \cdot\left(\frac{\eta_{2}}{\varrho}\right)^{-\frac{\varrho}{1-(\rho+\varrho)}} \cdot[1-(\rho+\varrho)]$. Hence, the proof is complete.

We also recall the following Gagliardo-Nirenberg's interpolation inequality, which will be used frequently in the proof of our main results. 
Lemma 2.5. [9] Let $l$ and $k$ be two integers satisfying $l \in[0, k)$. Suppose that $q, r \in[1, \infty]$, $p>0$ and $a \in\left[\frac{l}{k}, 1\right]$ such that

$$
\frac{1}{p}-\frac{l}{n}=a\left(\frac{1}{q}-\frac{k}{n}\right)+(1-a) \frac{1}{r}
$$

Then, for any $\phi \in W^{k, q}(\Omega) \cap L^{r}(\Omega)$, there exist two positive constants $c_{1}$ and $c_{2}$ depending only on $\Omega, q, k, r$ such that

$$
\left\|D^{l} \phi\right\|_{L^{p}(\Omega)} \leq c_{1}\left\|D^{k} \phi\right\|_{L^{q}(\Omega)}^{a}\|\phi\|_{L^{r}(\Omega)}^{1-a}+c_{2}\|\phi\|_{L^{r}(\Omega)}
$$

with the following exception: If $q \in(1, \infty)$ and $k-l-\frac{n}{q}$ is a non-negative integer, then 2.5 holds only for $a \in\left[\frac{l}{k}, 1\right)$. Here $D^{k} \phi$ is expressed as Fréchet derivative of order $k$. In particular, if $l=0, k=1$ and $q=2$, we deduce from (2.6)

$$
\|\phi\|_{L^{p}(\Omega)} \leq c_{1}\|\nabla \phi\|_{L^{2}(\Omega)}^{a}\|\phi\|_{L^{r}(\Omega)}^{1-a}+c_{2}\|\phi\|_{L^{r}(\Omega)},
$$

where $a \in(0,1)$ satisfying

$$
\frac{n}{p}=a\left(\frac{n}{2}-1\right)+\frac{n}{r}(1-a)
$$

Finally, we give a result referred to as a variation of maximal Sobolev regularity.

Lemma 2.6. 12, Lemma 3.1] Let $\sigma>1$. Consider the following evolution equation

$$
\begin{cases}Z_{t}=\Delta Z-Z+h, & (x, t) \in \Omega \times(0, T), \\ \frac{\partial Z}{\partial \nu}=0, & (x, t) \in \partial \Omega \times(0, T), \\ Z(x, 0)=Z_{0}(x), & x \in \Omega .\end{cases}
$$

Then for each $Z_{0} \in W^{2, \sigma}(\Omega)(\sigma>n)$ with $\left.\frac{\partial Z_{0}}{\partial \nu}\right|_{\partial \Omega}=0$ and any $h \in L^{\sigma}\left((0, T) ; L^{\sigma}(\Omega)\right)$, there exists a unique solution

$$
Z \in W^{1, \sigma}\left((0, T) ; L^{\sigma}(\Omega)\right) \cap L^{\sigma}\left((0, T) ; W^{2, \sigma}(\Omega)\right)
$$

Moreover, there exists positive constant $C_{\sigma}$, such that if $t_{0} \in[0, T), Z\left(\cdot, t_{0}\right) \in W^{2, \sigma}(\Omega)$ $(\sigma>n)$ with $\left.\frac{\partial Z\left(\cdot, t_{0}\right)}{\partial \nu}\right|_{\partial \Omega}=0$, then

$$
\int_{t_{0}}^{T} \int_{\Omega} e^{\frac{\sigma}{2} \tau}|\Delta Z|^{\sigma} \leq C_{\sigma} \int_{t_{0}}^{T} \int_{\Omega} e^{\frac{\sigma}{2} \tau}|h|^{\sigma}+C_{\sigma} e^{\frac{\sigma}{2} t_{0}}\left[\left\|Z\left(\cdot, t_{0}\right)\right\|_{L^{\sigma}}^{\sigma}+\left\|\Delta Z\left(\cdot, t_{0}\right)\right\|_{L^{\sigma}}^{\sigma}\right] .
$$

\section{Boundedness}

We divide this section into two subsections to prove the boundedness of the solution. 


\subsection{Boundedness without logistic source}

The goal of this subsection is to establish uniform-in-time boundedness for $\|u\|_{L^{p}(\Omega)}$ and $\|\omega\|_{L^{p}(\Omega)}$ as well as $\|\nabla v\|_{L^{q}(\Omega)}$ and $\|\nabla z\|_{L^{q}(\Omega)}$ for arbitrarily large $p$ and $q$.

In order to prove Theorem 1.1, we need some preparations. We first employ trace inequality, Hölder's inequality, Gagliardo-Nirenberg's inequality, Proposition 2.4 and Young's inequality to establish the following useful proposition.

Proposition 3.1. Let $n \geq 2$ and nonnegative initial data $\left(u_{0}, v_{0}, \omega_{0}, z_{0}\right)$ satisfy (1.5). Suppose D, S, $\widetilde{D}, \widetilde{S}$ and $g, \widetilde{g}$ fulfill (1.6) and (1.7) respectively. If $\gamma, \widetilde{\gamma} \in(0,1]$ and $\alpha_{1}=\widetilde{\alpha}_{1}$ as well as

$$
\alpha=\widetilde{\alpha}<\frac{2}{n} \quad \text { and } \quad \alpha+\max \{\beta+\gamma, \widetilde{\beta}+\widetilde{\gamma}\}<1+\frac{2}{n},
$$

then for all $p, q \in[1, \infty)$, there exists positive constant $C=C\left(p, q, \alpha, \alpha_{1}, \beta, \widetilde{\beta}, \gamma, \widetilde{\gamma}\right)$ such that

$$
\|u\|_{L^{p}(\Omega)},\|\omega\|_{L^{p}(\Omega)},\|\nabla v\|_{L^{q}(\Omega)},\|\nabla z\|_{L^{q}(\Omega)} \leq C \quad \text { for all } t \in\left(0, T_{\max }\right) .
$$

Proof. The Sobolev embedding theorem conveys to us that the lower order $\|u\|_{L^{p}(\Omega)}$ and $\|\omega\|_{L^{p}(\Omega)}$ as well as $\|\nabla v\|_{L^{q}(\Omega)}$ and $\|\nabla z\|_{L^{q}(\Omega)}$ can be controlled by the higher order $\|u\|_{L^{p}(\Omega)}$ and $\|\omega\|_{L^{p}(\Omega)}$ as well as $\|\nabla v\|_{L^{q}(\Omega)}$ and $\|\nabla z\|_{L^{q}(\Omega)}$ respectively. Therefore, we only need to prove that for sufficiently large numbers $p$ and $q$ there exists positive constant $C=$ $C\left(p, q, \alpha, \alpha_{1}, \beta, \widetilde{\beta}, \gamma, \widetilde{\gamma}\right)$ such that

$$
\|u\|_{L^{p}(\Omega)},\|\omega\|_{L^{p}(\Omega)},\|\nabla v\|_{L^{q}(\Omega)},\|\nabla z\|_{L^{q}(\Omega)} \leq C \quad \text { for all } t \in\left(0, T_{\max }\right) .
$$

To quantify sufficiently large numbers $p$ and $q$, for the sake of the proof we find the lower bounds $\bar{p}$ and $\bar{q}$ of numbers $p$ and $q$ respectively as follows:

Under the assumptions $\gamma, \widetilde{\gamma} \in(0,1]$ and $\alpha_{1}=\widetilde{\alpha}_{1}$ as well as $\alpha=\widetilde{\alpha}<\frac{2}{n}$ and $\alpha+$ $\max \{\beta+\gamma, \widetilde{\beta}+\widetilde{\gamma}\}<1+\frac{2}{n}$, we can fix $s \in\left[1, \frac{n}{(n \gamma-1)_{+}}\right) \cap\left[1, \frac{n}{(n \widetilde{\gamma}-1)_{+}}\right)$such that

$$
\gamma-\frac{1}{n}<\frac{1}{s}<1+\frac{1}{n}-(\alpha+\beta) \text { and } \widetilde{\gamma}-\frac{1}{n}<\frac{1}{s}<1+\frac{1}{n}-(\alpha+\widetilde{\beta}) \text {. }
$$

Next, we can pick some $a, \widetilde{a}, b, \widetilde{b}$ fulfilling

$$
a, \tilde{a} \in\left(1, \min \left\{\frac{n}{n-2}, \frac{s}{(s-2)_{+}}\right\}\right), \quad b>\max \left\{\frac{n}{2}, \frac{1}{2 \gamma}\right\}, \quad \widetilde{b}>\max \left\{\frac{n}{2}, \frac{1}{2 \widetilde{\gamma}}\right\} .
$$

Then, we can select $\bar{p}$ and $\bar{q}$ large enough satisfying

$$
\bar{p}>\max \left\{1,1+\alpha-\alpha_{1}, 1+\alpha+\frac{2}{s}\right\} \quad \text { and } \quad \bar{q}>1+\frac{s}{2}
$$


as well as

$$
\left\{\begin{array}{l}
\frac{n-2}{n}\left[1+\frac{2|\alpha+\beta-1|}{\bar{p}-\alpha}\right]<\frac{1}{a}<\bar{p}+\alpha+2 \beta-2, \\
\frac{n-2}{n}\left[1+\frac{2|\alpha+\widetilde{\beta}-1|}{\bar{p}-\alpha}\right]<\frac{1}{\widetilde{a}}<\bar{p}+\alpha+2 \widetilde{\beta}-2, \\
\frac{n-2}{n \bar{q}}<1-\frac{1}{a}, \quad \frac{n-2}{n \bar{q}}<1-\frac{1}{\widetilde{a}}, \\
\frac{n-2}{n} \cdot \frac{2 \gamma}{\bar{p}-\alpha}<\frac{1}{b}, \quad \frac{n-2}{n} \cdot \frac{2 \widetilde{\gamma}}{\bar{p}-\alpha}<\frac{1}{\widetilde{b}}, \\
\bar{q}<\frac{\bar{p}-\alpha}{2} s .
\end{array}\right.
$$

Finally, it is easy to check that

$$
\frac{n-2}{n}\left[1+\frac{2(\alpha+\beta-1)}{p-\alpha}\right]=\frac{n-2}{n} \cdot \frac{p+\alpha+2 \beta-2}{p-\alpha}<\frac{1}{a}<p+\alpha+2 \beta-2
$$

and

$$
\begin{gathered}
1-\frac{2}{s}<\frac{1}{a}<1-\frac{n-2}{n q}, \\
\frac{n-2}{n} \cdot \frac{2 \gamma}{p-\alpha}<\frac{1}{b}<\frac{2}{n}, \\
\frac{n-2}{n}\left[1+\frac{2(\alpha+\widetilde{\beta}-1)}{p-\alpha}\right]=\frac{n-2}{n} \cdot \frac{p+\alpha+2 \widetilde{\beta}-2}{p-\alpha}<\frac{1}{\widetilde{a}}<p+\alpha+2 \widetilde{\beta}-2, \\
1-\frac{2}{s}<\frac{1}{\widetilde{a}}<1-\frac{n-2}{n q}
\end{gathered}
$$

as well as

$$
\frac{n-2}{n} \cdot \frac{2 \widetilde{\gamma}}{p-\alpha}<\frac{1}{\widetilde{b}}<\frac{2}{n}
$$

hold for all $p \geq \bar{p}$ and $q \geq \bar{q}$. What we need to keep in mind is that when $p \geq \bar{p}$ and $q \geq \bar{q}$, (3.4) -3.9 hold, which will guarantee that all of $\theta, \delta, \bar{\theta}, \bar{\delta}, \widetilde{\theta}, \widetilde{\delta}, \widetilde{\bar{\theta}}, \widetilde{\bar{\delta}}$ in 3.22 -3.25) fall in the interval $(0,1)$.

With the above preparations at hand, we define

$$
\phi(\zeta)=\int_{0}^{\zeta} \int_{0}^{\rho} \frac{(1+\sigma)^{p-\alpha-2}}{D(\sigma)} d \sigma d \rho, \quad \psi(\zeta)=\int_{0}^{\zeta} \int_{0}^{\rho} \frac{(1+\sigma)^{p-\alpha-2}}{\widetilde{D}(\sigma)} d \sigma d \rho, \quad \zeta \geq 0 .
$$

It is easy to check that the assumption $p>1+\alpha-\alpha_{1}$ in 3.3 ensures that $\phi$ and $\psi$ are well-defined and nonnegative. Multiplying the first equation in (1.4) by $\phi(u)$ and integrating by parts over $\Omega$, we can deduce

$$
\begin{aligned}
\frac{d}{d t} \int_{\Omega} \phi(u) & =-\int_{\Omega} \phi^{\prime \prime}(u) D(u)|\nabla u|^{2}+\int_{\Omega} \phi^{\prime \prime}(u) S(u) \nabla u \cdot \nabla v \\
& =-\int_{\Omega}(1+u)^{p-\alpha-2}|\nabla u|^{2}+\int_{\Omega}(1+u)^{p-\alpha-2} \frac{S(u)}{D(u)} \nabla u \cdot \nabla v \\
& \leq-\int_{\Omega}(1+u)^{p-\alpha-2}|\nabla u|^{2}+\frac{s_{1}}{d_{0}} \int_{\Omega}(1+u)^{p+\beta-2}|\nabla u| \cdot|\nabla v| \\
& \leq-\frac{1}{2} \int_{\Omega}(1+u)^{p-\alpha-2}|\nabla u|^{2}+\frac{s_{1}^{2}}{2 d_{0}^{2}} \int_{\Omega}(1+u)^{p+\alpha+2 \beta-2}|\nabla v|^{2},
\end{aligned}
$$


where we have used Young's inequality in the last two inequalities. Rewriting (3.10) produces

$$
\frac{d}{d t} \int_{\Omega} \phi(u)+\frac{2}{(p-\alpha)^{2}} \int_{\Omega}\left|\nabla(1+u)^{\frac{p-\alpha}{2}}\right|^{2} \leq \frac{s_{1}^{2}}{2 d_{0}^{2}} \int_{\Omega}(1+u)^{p+\alpha+2 \beta-2}|\nabla v|^{2}
$$

A straightforward calculation shows $\triangle|\nabla v|^{2}=2\left|D^{2} v\right|^{2}+2 \nabla v \cdot \nabla \triangle v$. Applying the second equation in (1.4) and the pointwise estimate $|\triangle v|^{2} \leq n\left|D^{2} v\right|^{2}$, we can easily get

$$
\left(|\nabla v|^{2}\right)_{t}+\frac{2}{n}|\triangle v|^{2}+2|\nabla v|^{2} \leq \Delta|\nabla v|^{2}+2 \nabla v \cdot \nabla g(\omega)
$$

Multiplying $(3.12)$ by $|\nabla v|^{2(q-1)}$ and applying Lemma 2.3 , we can find some positive constant $C_{1}=C_{1}(q)$ such that

$$
\begin{aligned}
& \frac{1}{q} \frac{d}{d t} \int_{\Omega}|\nabla v|^{2 q}+\frac{2}{n} \int_{\Omega}|\nabla v|^{2(q-1)}|\Delta v|^{2}+2 \int_{\Omega}|\nabla v|^{2 q} \\
\leq & \int_{\Omega}|\nabla v|^{2(q-1)} \triangle|\nabla v|^{2}+2 \int_{\Omega}|\nabla v|^{2(q-1)} \nabla v \cdot \nabla g(\omega) \\
= & -\left.\left.(q-1) \int_{\Omega}|\nabla v|^{2(q-2)}|\nabla| \nabla v\right|^{2}\right|^{2}+\int_{\partial \Omega}|\nabla v|^{2(q-1)} \frac{\partial|\nabla v|^{2}}{\partial \nu} \\
& -2(q-1) \int_{\Omega}|\nabla v|^{2(q-2)} \nabla|\nabla v|^{2} \cdot \nabla v \cdot g(\omega)-2 \int_{\Omega}|\nabla v|^{2(q-1)} \Delta v \cdot g(\omega) \\
\leq & -\left.\left.\frac{q-1}{2} \int_{\Omega}|\nabla v|^{2(q-2)}|\nabla| \nabla v\right|^{2}\right|^{2}+2 \kappa_{\Omega} \int_{\partial \Omega}|\nabla v|^{2 q} \\
& +\frac{2}{n} \int_{\Omega}|\nabla v|^{2(q-1)}|\Delta v|^{2}+\left[2(q-1)+\frac{n}{2}\right] \int_{\Omega}|\nabla v|^{2(q-1)} g^{2}(\omega) \\
= & -\left.\left.\frac{2(q-1)}{q^{2}} \int_{\Omega}|\nabla| \nabla v\right|^{q}\right|^{2}+2 \kappa_{\Omega} \int_{\partial \Omega}|\nabla v|^{2 q} \\
& +\frac{2}{n} \int_{\Omega}|\nabla v|^{2(q-1)}|\Delta v|^{2}+\left[2(q-1)+\frac{n}{2}\right] \int_{\Omega}|\nabla v|^{2(q-1)} g^{2}(\omega) \\
\leq & -\left.\left.\frac{q-1}{q^{2}} \int_{\Omega}|\nabla| \nabla v\right|^{q}\right|^{2}+C_{1} \int_{\partial \Omega}|\nabla v|^{2 q} \\
& +\frac{2}{n} \int_{\Omega}|\nabla v|^{2(q-1)}|\triangle v|^{2}+\left[2(q-1)+\frac{n}{2}\right] \int_{\Omega}|\nabla v|^{2(q-1)} g^{2}(\omega)
\end{aligned}
$$

for all $t \in\left(0, T_{\max }\right)$, where we have used the trace inequality (see [37, Proposition 3.1] and [11, Propositions 4.22 and 4.24])

$$
2 \kappa_{\Omega}\|h\|_{L^{2}(\partial \Omega)}^{2} \leq \frac{q-1}{q^{2}}\|\nabla h\|_{L^{2}(\Omega)}^{2}+C_{1}\|h\|_{L^{2}(\Omega)}^{2} \quad \text { for all } h \in W^{1,2}(\Omega) .
$$


Collecting (3.13) and 1.7 leads to

$$
\begin{aligned}
& \frac{1}{q} \frac{d}{d t} \int_{\Omega}|\nabla v|^{2 q}+\left.\left.\frac{q-1}{q^{2}} \int_{\Omega}|\nabla| \nabla v\right|^{q}\right|^{2} \\
\leq & \lambda_{1}^{2}\left[2(q-1)+\frac{n}{2}\right] \int_{\Omega} \omega^{2 \gamma}|\nabla v|^{2(q-1)}+\left(C_{1}-2\right) \int_{\Omega}|\nabla v|^{2 q} \\
\leq & \lambda_{1}^{2}\left[2(q-1)+\frac{n}{2}\right] \int_{\Omega}(1+\omega)^{2 \gamma}|\nabla v|^{2(q-1)}+\left(C_{1}-2\right) \int_{\Omega}|\nabla v|^{2 q} .
\end{aligned}
$$

A linear combination (3.11) and (3.14) results in

$$
\begin{aligned}
& \frac{d}{d t} \int_{\Omega}\left\{\phi(u)+\frac{1}{q}|\nabla v|^{2 q}\right\}+\frac{2}{(p-\alpha)^{2}} \int_{\Omega}\left|\nabla(1+u)^{\frac{p-\alpha}{2}}\right|^{2}+\left.\left.\frac{q-1}{q^{2}} \int_{\Omega}|\nabla| \nabla v\right|^{q}\right|^{2} \\
\leq & C_{2} \int_{\Omega}(1+u)^{p+\alpha+2 \beta-2}|\nabla v|^{2}+C_{2} \int_{\Omega}|\nabla v|^{2 q}+C_{2} \int_{\Omega}(1+\omega)^{2 \gamma}|\nabla v|^{2(q-1)},
\end{aligned}
$$

where positive constants $C_{1}>2$ and $C_{2}=\max \left\{\frac{s_{1}^{2}}{2 d_{0}^{2}}, \lambda_{1}^{2}\left[2(q-1)+\frac{n}{2}\right], C_{1}-2\right\}$. Performing similar operations on the third and fourth equations of 1.4 produces

$$
\begin{aligned}
& \frac{d}{d t} \int_{\Omega}\left\{\psi(\omega)+\frac{1}{q}|\nabla z|^{2 q}\right\}+\frac{2}{(p-\alpha)^{2}} \int_{\Omega}\left|\nabla(1+\omega)^{\frac{p-\alpha}{2}}\right|^{2}+\left.\left.\frac{q-1}{q^{2}} \int_{\Omega}|\nabla| \nabla z\right|^{q}\right|^{2} \\
\leq & \widetilde{C}_{2} \int_{\Omega}(1+\omega)^{p+\alpha+2 \widetilde{\beta}-2}|\nabla z|^{2}+\widetilde{C}_{2} \int_{\Omega}|\nabla z|^{2 q}+\widetilde{C}_{2} \int_{\Omega}(1+u)^{2 \widetilde{\gamma}}|\nabla z|^{2(q-1)},
\end{aligned}
$$

where positive constants $\widetilde{C}_{2}=\max \left\{\frac{\widetilde{s}_{1}^{2}}{2 \widetilde{d}_{0}^{2}}, \widetilde{\lambda}_{1}^{2}\left[2(q-1)+\frac{n}{2}\right], \widetilde{C}_{1}-2\right\}$ and $\widetilde{C}_{1}>2$. A combination 3.15$)$ and $(3.16)$ yields

$$
\begin{aligned}
& \frac{d}{d t} \int_{\Omega}\left\{\phi(u)+\psi(\omega)+\frac{1}{q}|\nabla v|^{2 q}+\frac{1}{q}|\nabla z|^{2 q}\right\}+\frac{2}{(p-\alpha)^{2}} \int_{\Omega}\left|\nabla(1+u)^{\frac{p-\alpha}{2}}\right|^{2} \\
& +\frac{2}{(p-\alpha)^{2}} \int_{\Omega}\left|\nabla(1+\omega)^{\frac{p-\alpha}{2}}\right|^{2}+\left.\left.\frac{q-1}{q^{2}} \int_{\Omega}|\nabla| \nabla v\right|^{q}\right|^{2}+\left.\left.\frac{q-1}{q^{2}} \int_{\Omega}|\nabla| \nabla z\right|^{q}\right|^{2} \\
& \leq C_{2} \int_{\Omega}(1+u)^{p+\alpha+2 \beta-2}|\nabla v|^{2}+\widetilde{C}_{2} \int_{\Omega}(1+\omega)^{p+\alpha+2 \widetilde{\beta}-2}|\nabla z|^{2}+C_{2} \int_{\Omega}(1+\omega)^{2 \gamma}|\nabla v|^{2(q-1)} \\
& +\widetilde{C}_{2} \int_{\Omega}(1+u)^{2 \widetilde{\gamma}}|\nabla z|^{2(q-1)}+C_{2} \int_{\Omega}|\nabla v|^{2 q}+\widetilde{C}_{2} \int_{\Omega}|\nabla z|^{2 q} .
\end{aligned}
$$

In order to control the first four integrals on the right hand by the last four integrals on the left hand side, we use Hölder's inequality four times to estimate the integrals on the right hand side of 3.17 as follows

$$
\int_{\Omega}(1+u)^{p+\alpha+2 \beta-2}|\nabla v|^{2} \leq\left\{\int_{\Omega}(1+u)^{(p+\alpha+2 \beta-2) a}\right\}^{\frac{1}{a}} \cdot\left\{\int_{\Omega}|\nabla v|^{2 a^{\prime}}\right\}^{\frac{1}{a^{\prime}}}
$$




$$
\begin{aligned}
\int_{\Omega}(1+\omega)^{2 \gamma}|\nabla v|^{2(q-1)} & \leq\left\{\int_{\Omega}(1+\omega)^{2 \gamma b}\right\}^{\frac{1}{b}} \cdot\left\{\int_{\Omega}|\nabla v|^{2(q-1) b^{\prime}}\right\}^{\frac{1}{b^{\prime}}}, \\
\int_{\Omega}(1+\omega)^{p+\alpha+2 \widetilde{\beta}-2}|\nabla z|^{2} & \leq\left\{\int_{\Omega}(1+\omega)^{(p+\alpha+2 \widetilde{\beta}-2) \widetilde{a}}\right\}^{\frac{1}{\tilde{a}}} \cdot\left\{\int_{\Omega}|\nabla z|^{2 \widetilde{a}^{\prime}}\right\}^{\frac{1}{\widetilde{a}^{\prime}}}, \\
\int_{\Omega}(1+u)^{2 \widetilde{\gamma}}|\nabla z|^{2(q-1)} & \leq\left\{\int_{\Omega}(1+u)^{2 \widetilde{\gamma} \widetilde{b}}\right\}^{\frac{1}{\widetilde{b}}} \cdot\left\{\int_{\Omega}|\nabla z|^{2(q-1) \widetilde{b^{\prime}}}\right\}^{\frac{1}{\widetilde{b}^{\prime}}},
\end{aligned}
$$

where all of $a, b, \widetilde{a}, \widetilde{b}$ satisfy $(3.2)$. And then we get $a^{\prime}=\frac{a}{a-1}>1, b^{\prime}=\frac{b}{b-1}>1$, $\widetilde{a}^{\prime}=\frac{\widetilde{a}}{\tilde{a}-1}>1, \widetilde{b}^{\prime}=\frac{\widetilde{b}}{\tilde{b}-1}>1$. In view of Lemma 2.2 and 3.4 , applying the GagliardoNirenberg's inequality (2.7) results in

$$
\begin{aligned}
& \left\{\int_{\Omega}(1+u)^{(p+\alpha+2 \beta-2) a}\right\}^{\frac{1}{a}} \\
= & \left\|(1+u)^{\frac{p-\alpha}{2}}\right\|^{\frac{2(p+\alpha+2 \beta-2)}{p-\alpha}} L^{\frac{2 \alpha(p+\alpha+2 \beta-2)}{p-\alpha}}(\Omega) \\
\leq & C_{3}\left\{\left\|\nabla(1+u)^{\frac{p-\alpha}{2}}\right\|_{L^{2}(\Omega)}^{\frac{2(p+\alpha+2 \beta-2) \theta}{p-\alpha}}\left\|(1+u)^{\frac{p-\alpha}{2}}\right\|_{L^{\frac{2(p+\alpha+2 \beta-2)(1-\theta)}{p-\alpha}}(\Omega)}^{\frac{2(p)}{p-\alpha}}+\left\|(1+u)^{\frac{p-\alpha}{2}}\right\|_{L^{\frac{2(p+\alpha+\alpha}{p-\alpha}}(\Omega)}^{\frac{2}{p-\alpha}}\right\} \\
\leq & C_{4}\left\{\int_{\Omega}\left|\nabla(1+u)^{\frac{p-\alpha}{2}}\right|^{2}\right\}^{\frac{(p+\alpha+2 \beta-2) \theta}{p-\alpha}}+C_{4},
\end{aligned}
$$

where positive constants $C_{3}=C_{3}(p, \alpha)$ and $C_{4}=C_{4}(p, \alpha, \beta)$; the assumptions $p>1$ and (3.4) guarantee $\theta=\frac{\frac{p-\alpha}{2}-\frac{p-\alpha}{2 a(p+\alpha+2 \beta-2)}}{\frac{1}{n}-\frac{1}{2}+\frac{p-\alpha}{2}} \in(0,1)$. We use Lemma 2.2 and the GagliardoNirenberg's inequality 2.7 once again to obtain

$$
\begin{aligned}
\left\{\int_{\Omega}|\nabla v|^{2 a^{\prime}}\right\}^{\frac{1}{a^{\prime}}} & =\left\||\nabla v|^{q}\right\|_{L^{\frac{2 a^{\prime}}{q}}(\Omega)}^{\frac{2}{q}} \\
& \leq C_{5}\left\{\left\|\nabla|\nabla v|^{q}\right\|_{L^{2}(\Omega)}^{\frac{2 \delta}{q}}\left\||\nabla v|^{q}\right\|_{L^{\frac{s^{q}}{q}(\Omega)}}^{\frac{2(1-\delta)}{q}}+\left\||\nabla v|^{q}\right\|_{L^{\frac{2}{q}}(\Omega)}^{\frac{2}{q}}\right\} \\
& \leq C_{6}\left\{\left.\left.\int_{\Omega}|\nabla| \nabla v\right|^{q}\right|^{2}\right\}^{\frac{\delta}{q}}+C_{6},
\end{aligned}
$$

where positive constants $C_{5}=C_{5}(q, s)$ and $C_{6}=C_{6}(q, s, a, \gamma)$; the assumptions 3.5 and $q>1+\frac{s}{2}$ in (3.3) warrant $\delta=\frac{\frac{q}{s}+\frac{q}{2 a}-\frac{q}{2}}{\frac{1}{n}-\frac{1}{2}+\frac{q}{s}} \in(0,1)$. Inserting 3.20) and 3.21 into (3.18), we can find some positive constant $C_{7}=C_{7}(p, q, \alpha, \beta, a, s, \gamma)$ fulfilling

$$
\begin{aligned}
& C_{2} \int_{\Omega}(1+u)^{p+\alpha+2 \beta-2}|\nabla v|^{2} \\
\leq & C_{7}\left\{\left[\int_{\Omega}\left|\nabla(1+u)^{\frac{p-\alpha}{2}}\right|^{2}\right]^{\frac{(p+\alpha+2 \beta-2) \theta}{p-\alpha}}\left[\left.\left.\int_{\Omega}|\nabla| \nabla v\right|^{q}\right|^{2}\right]^{\frac{\delta}{q}}\right. \\
+ & {\left.\left[\int_{\Omega}\left|\nabla(1+u)^{\frac{p-\alpha}{2}}\right|^{2}\right]^{\frac{(p+\alpha+2 \beta-2) \theta}{p-\alpha}}+\left[\left.\left.\int_{\Omega}|\nabla| \nabla v\right|^{q}\right|^{2}\right]^{\frac{\delta}{q}}+1\right\} . }
\end{aligned}
$$


Similarly, (3.6) and Lemma 2.2 along with the Gagliardo-Nirenberg's inequality (2.7) indicate that

$$
\left\{\int_{\Omega}(1+\omega)^{2 \gamma b}\right\}^{\frac{1}{b}}=\left\|(1+\omega)^{\frac{p-\alpha}{2}}\right\|_{L^{\frac{4 \gamma b}{p-\alpha}}}^{\frac{4 \gamma}{p-\alpha}} \leq C_{8}\left\{\int_{\Omega}\left|\nabla(1+\omega)^{\frac{p-\alpha}{2}}\right|^{2}\right\}^{\frac{2 \gamma}{p-\alpha} \bar{\theta}}+C_{8}
$$

and

$$
\left\{\int_{\Omega}|\nabla v|^{2(q-1) b^{\prime}}\right\}^{\frac{1}{b^{\prime}}}=\left\||\nabla v|^{q}\right\|_{L^{\frac{2(q-1) b^{\prime}}{q}(\Omega)}}^{\frac{2(q-1)}{q}} \leq C_{9}\left\{\left.\left.\int_{\Omega}|\nabla| \nabla v\right|^{q}\right|^{2}\right\}^{\frac{(q-1) \bar{\delta}}{q}}+C_{9}
$$

where the positive constants $C_{8}=C_{8}(p, \alpha, b, \gamma)$ and $C_{9}=C_{9}(q, s, b, \gamma) ; p>1$ in (3.3) and $b>\frac{1}{2 \gamma}$ in $(3.2)$ as well as $\frac{n-2}{n} \cdot \frac{2 \gamma}{p-\alpha}<\frac{1}{b}$ in $(3.6)$ ensure $\bar{\theta}=\frac{\frac{p-\alpha}{2}-\frac{p-\alpha}{4 \gamma b}}{\frac{1}{n}-\frac{1}{2}+\frac{p-\alpha}{2}} \in(0,1)$; (3.8) warrants $\frac{1}{s}>\frac{n-2}{2 n q} ; q>1+\frac{s}{2}$ in (3.3) guarantees $s<\frac{2 b(q-1)}{b-1}$; in the same way, $\frac{1}{s}>\frac{n-2}{2 n q}$ and $s<\frac{2 b(q-1)}{b-1}$ as well as $\frac{1}{b}<\frac{2}{n} \leq \frac{2}{n}+\frac{1}{q}\left(1-\frac{2}{n}\right)$ ensure $\bar{\delta}=\frac{\frac{q}{s}+\frac{q}{2(q-1) b}-\frac{q}{2(q-1)}}{\frac{1}{n}-\frac{1}{2}+\frac{q}{s}} \in(0,1)$. Inserting the above two inequalities into 3.19 gives us

$$
\begin{aligned}
& C_{2} \int_{\Omega}(1+\omega)^{2 \gamma}|\nabla v|^{2(q-1)} \\
\leq & C_{10}\left\{\left[\int_{\Omega}\left|\nabla(1+\omega)^{\frac{p-\alpha}{2}}\right|^{2}\right]^{\frac{2 \gamma \bar{\theta}}{p-\alpha}}\left[\left.\left.\int_{\Omega}|\nabla| \nabla v\right|^{q}\right|^{2}\right]^{\frac{(q-1) \bar{\delta}}{q}}\right. \\
+ & {\left.\left[\int_{\Omega}\left|\nabla(1+\omega)^{\frac{p-\alpha}{2}}\right|^{2}\right]^{\frac{2 \gamma \bar{\theta}}{p-\alpha}}+\left[\left.\left.\int_{\Omega}|\nabla| \nabla v\right|^{q}\right|^{2}\right]^{\frac{(q-1) \bar{\delta}}{q}}+1\right\}, }
\end{aligned}
$$

where positive constant $C_{10}=C_{10}(p, \alpha, b, \gamma, q, s)$. In precisely the same manner, we get there exist positive constants $\widetilde{C}_{7}$ and $\widetilde{C}_{10}$ such that

$$
\begin{aligned}
& \widetilde{C}_{2} \int_{\Omega}(1+\omega)^{p+\alpha+2 \widetilde{\beta}-2}|\nabla z|^{2} \\
& \leq \widetilde{C}_{7}\left\{\left[\int_{\Omega}\left|\nabla(1+\omega)^{\frac{p-\alpha}{2}}\right|^{2}\right]^{\frac{(p+\alpha+2 \widetilde{\beta}-2) \widetilde{\theta}}{p-\alpha}}\left[\left.\left.\int_{\Omega}|\nabla| \nabla z\right|^{q}\right|^{2}\right]^{\frac{\widetilde{\delta}}{q}}\right. \\
&\left.\quad+\left[\int_{\Omega}\left|\nabla(1+\omega)^{\frac{p-\alpha}{2}}\right|^{2}\right]^{\frac{(p+\alpha+2 \widetilde{\beta}-2) \widetilde{\theta}}{p-\alpha}}+\left[\left.\left.\int_{\Omega}|\nabla| \nabla z\right|^{q}\right|^{2}\right]^{\frac{\widetilde{\delta}}{q}}+1\right\}
\end{aligned}
$$

as well as

$$
\begin{aligned}
& \widetilde{C}_{2} \int_{\Omega}(1+u)^{2 \widetilde{\gamma}}|\nabla z|^{2(q-1)} \\
& \leq \widetilde{C}_{10}\left\{\left[\int_{\Omega}\left|\nabla(1+u)^{\frac{p-\alpha}{2}}\right|^{2}\right]^{\frac{2 \tilde{\gamma} \tilde{\bar{\theta}}}{p-\alpha}}\left[\left.\left.\int_{\Omega}|\nabla| \nabla z\right|^{q}\right|^{2}\right]^{\frac{(q-1) \tilde{\bar{\delta}}}{q}}\right. \\
&\left.+\left[\int_{\Omega}\left|\nabla(1+u)^{\frac{p-\alpha}{2}}\right|^{2}\right]^{\frac{2 \tilde{\gamma} \bar{\theta}}{p-\alpha}}+\left[\left.\left.\int_{\Omega}|\nabla| \nabla z\right|^{q}\right|^{2}\right]^{\frac{(q-1) \tilde{\bar{\delta}}}{q}}+1\right\} .
\end{aligned}
$$


Here positive constant $\widetilde{C}_{10}=\widetilde{C}_{10}(p, \alpha, \widetilde{b}, \widetilde{\gamma}, q, s) ; 3.7$ and $p>1$ in 3.3 ensure $\tilde{\theta}=$ $\frac{\frac{p-\alpha}{2}-\frac{p-\alpha}{2 \widetilde{a}(p+\alpha+2 \widetilde{\beta}-2)}}{\frac{1}{n}-\frac{1}{2}+\frac{p-\alpha}{2}} \in(0,1) ; 3.8$ and $q>1+\frac{s}{2}$ in $(3.3)$ guarantee $\widetilde{\delta}=\frac{\frac{q}{s}+\frac{q}{2 \widetilde{a}}-\frac{q}{2}}{\frac{1}{n}-\frac{1}{2}+\frac{q}{s}} \in(0,1) ; p>1$ in $(3.3)$ and $\widetilde{b}>\frac{1}{2 \widetilde{\gamma}}$ in $(3.2)$ as well as $\frac{n-2}{n} \cdot \frac{2 \widetilde{\gamma}}{p-\alpha}<\frac{1}{\widetilde{b}}$ in 3.9$)$ warrant $\widetilde{\bar{\theta}}=\frac{\frac{p-\alpha}{2}-\frac{p-\alpha}{4 \widetilde{\gamma} \tilde{b}}}{\frac{1}{n}-\frac{1}{2}+\frac{p-\alpha}{2}} \in(0,1)$; (3.8) warrants $\frac{1}{s}>\frac{n-2}{2 n q} ; q>1+\frac{s}{2}$ in 3.3 guarantees $s<\frac{2 \widetilde{b}(q-1)}{\widetilde{b}-1} ; \frac{1}{s}>\frac{n-2}{2 n q}$ and $s<\frac{2 \widetilde{b}(q-1)}{\widetilde{b}-1}$ as well as $\frac{1}{\widetilde{b}}<\frac{2}{n} \leq \frac{2}{n}+\frac{1}{q}\left(1-\frac{2}{n}\right)$ in 3.9 ensure $\widetilde{\widetilde{\delta}}=\frac{\frac{q}{s}+\frac{q}{2(q-1) \widetilde{b}}-\frac{q}{2(q-1)}}{\frac{1}{n}-\frac{1}{2}+\frac{q}{s}} \in(0,1)$.

Next, if the following four inequalities hold

$$
\begin{array}{ll}
\frac{p+\alpha+2 \beta-2}{p-\alpha} \theta+\frac{\delta}{q}<1, & \frac{2 \gamma \bar{\theta}}{p-\alpha}+\frac{q-1}{q} \bar{\delta}<1, \\
\frac{p+\alpha+2 \widetilde{\beta}-2}{p-\alpha} \widetilde{\theta}+\frac{\widetilde{\delta}}{q}<1, & \frac{2 \widetilde{\gamma} \widetilde{\bar{\theta}}}{p-\alpha}+\frac{q-1}{q} \widetilde{\bar{\delta}}<1,
\end{array}
$$

then applying Young's inequality to 3.17 , we can find some positive constant $C_{16}$ such that

$$
\frac{d}{d t} \int_{\Omega}\left\{\phi(u)+\psi(\omega)+\frac{1}{q}|\nabla v|^{2 q}+\frac{1}{q}|\nabla z|^{2 q}\right\}+\int_{\Omega}\left\{\phi(u)+\psi(\omega)+\frac{1}{q}|\nabla v|^{2 q}+\frac{1}{q}|\nabla z|^{2 q}\right\} \leq C_{16} .
$$

To this end, we define $H_{i}(q)(i=1,2,3,4)$ as follows

$$
\begin{aligned}
& H_{1}(q)=\frac{p+\alpha+2 \beta-2}{p-\alpha} \theta+\frac{\delta}{q}=\frac{\frac{p+\alpha+2 \beta-2}{2}-\frac{1}{2 a}}{\frac{1}{n}-\frac{1}{2}+\frac{p-\alpha}{2}}+\frac{\frac{1}{s}+\frac{1}{2 a}-\frac{1}{2}}{\frac{1}{n}-\frac{1}{2}+\frac{q}{s}}, \\
& H_{2}(q)=\frac{2 \gamma \bar{\theta}}{p-\alpha}+\frac{q-1}{q} \bar{\delta}=\frac{\gamma-\frac{1}{2 b}}{\frac{1}{n}-\frac{1}{2}+\frac{p-\alpha}{2}}+\frac{\frac{q-1}{s}+\frac{1}{2 b}-\frac{1}{2}}{\frac{1}{n}-\frac{1}{2}+\frac{q}{s}}, \\
& H_{3}(q)=\frac{p+\alpha+2 \widetilde{\beta}-2}{p-\alpha} \widetilde{\theta}+\frac{\widetilde{\delta}}{q}=\frac{\frac{p+\alpha+2 \widetilde{\beta}-2}{2}-\frac{1}{2 \widetilde{a}}}{\frac{1}{n}-\frac{1}{2}+\frac{\frac{p-\alpha}{2}}{2}+\frac{1}{2 \widetilde{a}}-\frac{1}{2}} \frac{\frac{1}{n}-\frac{1}{2}+\frac{q}{s}}{2} \\
& H_{4}(q)=\frac{2 \widetilde{\gamma} \tilde{\bar{\theta}}}{p-\alpha}+\frac{q-1}{q} \widetilde{\bar{\delta}}=\frac{\widetilde{\gamma}-\frac{1}{2 \widetilde{b}}}{\frac{1}{n}-\frac{1}{2}+\frac{p-\alpha}{2}}+\frac{\frac{1}{2 \widetilde{b}}-\frac{1}{2}}{\frac{1}{n}-\frac{1}{2}+\frac{q}{s}} .
\end{aligned}
$$

Let $q(p)=\frac{p-\alpha}{2} s$, then

$$
H_{1}(q(p))=\frac{\frac{p+\alpha+2 \beta-2}{2}-\frac{1}{2 a}}{\frac{1}{n}-\frac{1}{2}+\frac{p-\alpha}{2}}+\frac{\frac{1}{s}+\frac{1}{2 a}-\frac{1}{2}}{\frac{1}{n}-\frac{1}{2}+\frac{p-\alpha}{2}}=\frac{\frac{p+\alpha+2 \beta-2}{2}-\frac{1}{2}+\frac{1}{s}}{\frac{1}{n}-\frac{1}{2}+\frac{p-\alpha}{2}} .
$$

Thanks to the assumption $a<\frac{s}{(s-2)_{+}}$in 3.2 , we derive

$$
(p+\alpha+2 \beta-2)-1+\frac{2}{s}>(\bar{p}+\alpha+2 \beta-2)-1+\frac{2}{s}>\frac{1}{a}-1+\frac{2}{s}>0,
$$

hence, $\frac{p+\alpha+2 \beta-2}{2}-\frac{1}{2}+\frac{1}{s}>0$. From (3.3), we deduce $p>\bar{p}>1+\alpha+\frac{2}{s}>1+\alpha-\frac{2}{n}$, which implies $\frac{1}{n}-\frac{1}{2}+\frac{p-\alpha}{2}>0$. It is easy to verify that $\frac{1}{s}<1+\frac{1}{n}-(\alpha+\beta)$ in 3.1 ensures $H_{1}(q(p))<1$. Similarly, we will obtain $\frac{1}{s}>\gamma-\frac{1}{n}$ in 3.1 guarantees $H_{2}(q(p))<1$; 
$\frac{1}{s}<1+\frac{1}{n}-(\alpha+\widetilde{\beta})$ in 3.1 warrants $H_{3}(q(p))<1 ; \frac{1}{s}>\widetilde{\gamma}-\frac{1}{n}$ in 3.1 guarantees $H_{4}(q(p))<1$.

Similar to the proof in 37, by a continuity argument, for any $p \geq \bar{p}$, there exists $q \in[\bar{q}, q(p))$ close to $q(p)$ satisfying $H_{i}(q)<1(i=1,2,3,4)$, which together with the fact $q(p) \rightarrow \infty$ as $p \rightarrow \infty$ guarantees (3.26) for all $p \geq \bar{p}$ and $q \geq \bar{q}$.

We define the following parameters

$$
\begin{aligned}
\epsilon_{1} & =\frac{1}{2\left(C_{7}+\widetilde{C}_{10}\right)(p-\alpha)^{2}}, & \epsilon_{2} & =\frac{q-1}{4\left(C_{7}+C_{10}\right) q^{2}}, \\
\epsilon_{3} & =\frac{1}{2\left(\widetilde{C}_{7}+C_{10}\right)(p-\alpha)^{2}}, & \epsilon_{4} & =\frac{q-1}{4\left(\widetilde{C}_{7}+\widetilde{C}_{10}\right) q^{2}} .
\end{aligned}
$$

With $H_{1}(q)<1$ at hand, we see the exponent $\frac{p+\alpha+2 \beta-2}{p-\alpha} \theta \in(0,1)$ and $\frac{\delta}{q} \in(0,1)$ as well as $\frac{p+\alpha+2 \beta-2}{p-\alpha} \theta+\frac{\delta}{q}<1$. Applying Proposition 2.4 to the first term on the right-hand side of 3.22 produces

$$
\begin{aligned}
& C_{7}\left\{\int_{\Omega}\left|\nabla(1+u)^{\frac{p-\alpha}{2}}\right|^{2}\right\}^{\frac{(p+\alpha+2 \beta-2) \theta}{p-\alpha}} \cdot\left\{\left.\left.\int_{\Omega}|\nabla| \nabla v\right|^{q}\right|^{2}\right\}^{\frac{\delta}{q}} \\
\leq & C_{7}\left\{\epsilon_{1} \int_{\Omega}\left|\nabla(1+u)^{\frac{p-\alpha}{2}}\right|^{2}+\left.\left.\epsilon_{2} \int_{\Omega}|\nabla| \nabla v\right|^{q}\right|^{2}\right\}+\overline{\bar{C}}_{1} .
\end{aligned}
$$

Using Young's inequality to the second and third terms on the right-hand side of (3.22) gives us

$$
\left\{\int_{\Omega}\left|\nabla(1+u)^{\frac{p-\alpha}{2}}\right|^{2}\right\}^{\frac{(p+\alpha+2 \beta-2) \theta}{p-\alpha}} \leq \epsilon_{1} \int_{\Omega}\left|\nabla(1+u)^{\frac{p-\alpha}{2}}\right|^{2}+\widetilde{\widetilde{C}}_{1}
$$

and

$$
\left\{\left.\left.\int_{\Omega}|\nabla| \nabla v\right|^{q}\right|^{2}\right\}^{\frac{\delta}{q}} \leq\left.\left.\epsilon_{2} \int_{\Omega}|\nabla| \nabla v\right|^{q}\right|^{2}+\widehat{C}_{1} .
$$

Here all of $\overline{\bar{C}}_{1}, \widetilde{\widetilde{C}}_{1}$ and $\widehat{C}_{1}$ are positive constants. Repeating the process over and over again for 3.23 - 3.25), and inserting these results into 3.17), a straightforward calculation shows there exist positive constants $C_{i}^{\prime}(i=1,2,3,4)$ such that

$$
\begin{aligned}
& \quad \frac{d}{d t} \int_{\Omega}\left\{\phi(u)+\psi(\omega)+\frac{1}{q}|\nabla v|^{2 q}+\frac{1}{q}|\nabla z|^{2 q}\right\}+\frac{2}{(p-\alpha)^{2}} \int_{\Omega}\left|\nabla(1+u)^{\frac{p-\alpha}{2}}\right|^{2} \\
& +\frac{2}{(p-\alpha)^{2}} \int_{\Omega}\left|\nabla(1+\omega)^{\frac{p-\alpha}{2}}\right|^{2}+\left.\left.\frac{q-1}{q^{2}} \int_{\Omega}|\nabla| \nabla v\right|^{q}\right|^{2}+\left.\left.\frac{q-1}{q^{2}} \int_{\Omega}|\nabla| \nabla z\right|^{q}\right|^{2} \\
& \leq C_{7}\left[\epsilon_{1} \int_{\Omega}\left|\nabla(1+u)^{\frac{p-\alpha}{2}}\right|^{2}+\left.\left.\epsilon_{2} \int_{\Omega}|\nabla| \nabla v\right|^{q}\right|^{2}+\epsilon_{1} \int_{\Omega}\left|\nabla(1+u)^{\frac{p-\alpha}{2}}\right|^{2}+\left.\left.\epsilon_{2} \int_{\Omega}|\nabla| \nabla v\right|^{q}\right|^{2}+C_{1}^{\prime}\right] \\
& +C_{10}\left[\epsilon_{3} \int_{\Omega}\left|\nabla(1+\omega)^{\frac{p-\alpha}{2}}\right|^{2}+\left.\left.\epsilon_{2} \int_{\Omega}|\nabla| \nabla v\right|^{q}\right|^{2}+\epsilon_{3} \int_{\Omega}\left|\nabla(1+\omega)^{\frac{p-\alpha}{2}}\right|^{2}+\left.\left.\epsilon_{2} \int_{\Omega}|\nabla| \nabla v\right|^{q}\right|^{2}+C_{2}^{\prime}\right] \\
& +\widetilde{C}_{7}\left[\epsilon_{3} \int_{\Omega}\left|\nabla(1+\omega)^{\frac{p-\alpha}{2}}\right|^{2}+\left.\left.\epsilon_{4} \int_{\Omega}|\nabla| \nabla z\right|^{q}\right|^{2}+\epsilon_{3} \int_{\Omega}\left|\nabla(1+\omega)^{\frac{p-\alpha}{2}}\right|^{2}+\left.\left.\epsilon_{4} \int_{\Omega}|\nabla| \nabla z\right|^{q}\right|^{2}+C_{3}^{\prime}\right]
\end{aligned}
$$




$$
\begin{aligned}
& +\widetilde{C}_{10}\left[\epsilon_{1} \int_{\Omega}\left|\nabla(1+u)^{\frac{p-\alpha}{2}}\right|^{2}+\left.\left.\epsilon_{4} \int_{\Omega}|\nabla| \nabla z\right|^{q}\right|^{2}+\epsilon_{1} \int_{\Omega}\left|\nabla(1+u)^{\frac{p-\alpha}{2}}\right|^{2}+\left.\left.\epsilon_{4} \int_{\Omega}|\nabla| \nabla z\right|^{q}\right|^{2}+C_{4}^{\prime}\right] \\
& +C_{2} \int_{\Omega}|\nabla v|^{2 q}+\widetilde{C}_{2} \int_{\Omega}|\nabla z|^{2 q} .
\end{aligned}
$$

Meanwhile, a simple rearrangement leads to there exists some positive constant $C_{11}$ such that

$$
\begin{aligned}
& \frac{d}{d t} \int_{\Omega}\left\{\phi(u)+\psi(\omega)+\frac{1}{q}|\nabla v|^{2 q}+\frac{1}{q}|\nabla z|^{2 q}\right\} \\
& +\frac{1}{(p-\alpha)^{2}} \int_{\Omega}\left|\nabla(1+u)^{\frac{p-\alpha}{2}}\right|^{2}+\frac{1}{(p-\alpha)^{2}} \int_{\Omega}\left|\nabla(1+\omega)^{\frac{p-\alpha}{2}}\right|^{2} \\
& +\left.\left.\frac{q-1}{2 q^{2}} \int_{\Omega}|\nabla| \nabla v\right|^{q}\right|^{2}+\left.\left.\frac{q-1}{2 q^{2}} \int_{\Omega}|\nabla| \nabla z\right|^{q}\right|^{2} \\
& \leq C_{2} \int_{\Omega}|\nabla v|^{2 q}+\widetilde{C}_{2} \int_{\Omega}|\nabla z|^{2 q}+C_{11} .
\end{aligned}
$$

In view of the assumption $\alpha<\frac{2}{n}$, we obtain $\frac{p \sigma}{p-\alpha}<1$, and then there exists some positive constant $C_{13}$ such that

$$
\begin{aligned}
\int_{\Omega} \phi(u) & \leq \frac{1}{d_{0} p(p-1)} \int_{\Omega}(1+u)^{p}=\frac{1}{d_{0} p(p-1)}\left\|(1+u)^{\frac{p-\alpha}{2}}\right\|_{L^{\frac{2 p}{p-\alpha}}(\Omega)}^{\frac{2 p}{p-\alpha}} \\
& \leq C_{12}\left\{\int_{\Omega}\left|\nabla(1+u)^{\frac{p-\alpha}{2}}\right|^{2}\right\}^{\frac{p \sigma}{p-\alpha}}+C_{12} \\
& \leq \frac{1}{(p-\alpha)^{2}} \int_{\Omega}\left|\nabla(1+u)^{\frac{p-\alpha}{2}}\right|^{2}+C_{13}
\end{aligned}
$$

where $\sigma=\frac{\frac{p-\alpha}{2}-\frac{p-\alpha}{2 p}}{\frac{1}{n}-\frac{1}{2}+\frac{p-\alpha}{2}} \in(0,1)$ and positive constants $C_{12}=C_{12}(p, \alpha)$ as well as we have applied Young's inequality to the integral $\left[\int_{\Omega}\left|\nabla(1+u)^{\frac{p-\alpha}{2}}\right|^{2}\right]^{\frac{p \sigma}{p-\alpha}}$. Following the same argument as $u(x, t)$, we obtain there exists some positive constant $\widetilde{C}_{13}$ such that

$$
\int_{\Omega} \psi(\omega) \leq \frac{1}{(p-\alpha)^{2}} \int_{\Omega}\left|\nabla(1+\omega)^{\frac{p-\alpha}{2}}\right|^{2}+\widetilde{C}_{13}
$$

Applying the Gagliardo-Nirenberg's inequality (2.7) once again, we get there exist positive constants $C_{14}$ and $C_{15}$ such that

$$
\left(\frac{1}{q}+C_{2}\right) \int_{\Omega}|\nabla v|^{2 q} \leq C_{14}\left[\left.\left.\int_{\Omega}|\nabla| \nabla v\right|^{q}\right|^{2}\right]^{\bar{\sigma}}+C_{14} \leq\left.\left.\frac{q-1}{2 q^{2}} \int_{\Omega}|\nabla| \nabla v\right|^{q}\right|^{2}+C_{15}
$$

where $\bar{\sigma}=\frac{\frac{q}{s}-\frac{1}{2}}{\frac{1}{n}-\frac{1}{2}+\frac{q}{s}} \in(0,1)$. Following the same argument as $v(x, t)$, we deduce there exists positive constant $\widetilde{C}_{15}$ such that

$$
\left(\frac{1}{q}+\widetilde{C}_{2}\right) \int_{\Omega}|\nabla z|^{2 q} \leq\left.\left.\frac{q-1}{2 q^{2}} \int_{\Omega}|\nabla| \nabla z\right|^{q}\right|^{2}+\widetilde{C}_{15}
$$


Collecting (3.27)-(3.31), a simple rearrangement leads to

$$
\frac{d}{d t} \int_{\Omega}\left\{\phi(u)+\psi(\omega)+\frac{1}{q}|\nabla v|^{2 q}+\frac{1}{q}|\nabla z|^{2 q}\right\}+\int_{\Omega}\left\{\phi(u)+\psi(\omega)+\frac{1}{q}|\nabla v|^{2 q}+\frac{1}{q}|\nabla z|^{2 q}\right\} \leq C_{16},
$$

where positive constant $C_{16}=C_{11}+C_{13}+\widetilde{C}_{13}+C_{15}+\widetilde{C}_{15}$. By an ODE comparison argument, we derive

$$
\begin{aligned}
& \int_{\Omega} \phi(u)+\int_{\Omega} \psi(\omega)+\frac{1}{q} \int_{\Omega}|\nabla v|^{2 q}+\frac{1}{q} \int_{\Omega}|\nabla z|^{2 q} \\
\leq & \max \left\{\int_{\Omega} \phi\left(u_{0}\right)+\int_{\Omega} \psi\left(\omega_{0}\right)+\frac{1}{q} \int_{\Omega}\left|\nabla v_{0}\right|^{2 q}+\frac{1}{q} \int_{\Omega}\left|\nabla z_{0}\right|^{2 q}, C_{16}\right\} .
\end{aligned}
$$

Obviously, with the definition of $\phi(u)$ and $\psi(\omega)$ at hand, we obtain there exist positive constants $C_{17}$ and $\widetilde{C}_{17}$ such that

$$
(1+u)^{p+\alpha_{1}-\alpha} \leq C_{17}[\phi(u)+u+1] \quad \text { and } \quad(1+\omega)^{p+\alpha_{1}-\alpha} \leq \widetilde{C}_{17}[\psi(\omega)+\omega+1] .
$$

And then, we can find some positive constant $C_{18}$ satisfying

$$
\int_{\Omega}(1+u)^{p+\alpha_{1}-\alpha} \leq C_{18}, \quad \int_{\Omega}(1+\omega)^{p+\alpha_{1}-\alpha} \leq C_{18}, \quad \int_{\Omega}|\nabla v|^{2 q} \leq C_{18}, \quad \int_{\Omega}|\nabla z|^{2 q} \leq C_{18}
$$

for all $t \in\left(0, T_{\max }\right)$. Substituting $p$ with $p+\alpha-\alpha_{1}$ produces

$$
\sup _{t \in\left(0, T_{\max }\right)} \int_{\Omega}(1+u)^{p}<\infty \text { and } \sup _{t \in\left(0, T_{\max }\right)} \int_{\Omega}(1+\omega)^{p}<\infty .
$$

Using the Sobolev embedding $L^{2 q}(\Omega) \hookrightarrow L^{2 q-1}(\Omega)$ leads to

$$
\sup _{t \in\left(0, T_{\max }\right)} \int_{\Omega}|\nabla v|^{2 q-1}<\infty \text { and } \sup _{t \in\left(0, T_{\max }\right)} \int_{\Omega}|\nabla z|^{2 q-1}<\infty .
$$

As summarized above, we arrive at the desired estimate.

Now, we are in the position to complete the proof of Theorem 1.1, our proof reads as follows.

Proof of Theorem 1.1. We first take $p_{0}>\max \left\{1,1+\alpha-\alpha_{1}, \beta q_{1}\right\}$ large enough fulfilling (A.8)-(A.10) in [38] (Here, $q_{1}$ is chosen as follows). With Proposition 3.1 at hand, we can find some positive constant $C_{19}=C_{19}(\beta, \gamma, \widetilde{\gamma})$ satisfying

$$
\sup _{t \in\left(0, T_{\max }\right)}\|u\|_{L^{p_{0}(\Omega)}} \leq C_{19}, \quad \sup _{t \in\left(0, T_{\max }\right)}\|\omega\|_{L^{p_{0}(\Omega)}} \leq C_{19}
$$

Next, Choosing $q_{1}>n+2$ such that

$$
S(u) \nabla v \in L^{\infty}\left(\left(0, T_{\max }\right), L^{q_{1}}(\Omega)\right), \quad \widetilde{S}(\omega) \nabla z \in L^{\infty}\left(\left(0, T_{\max }\right), L^{q_{1}}(\Omega)\right) .
$$


In fact, when $\beta \leq 0$, we will see $S(u) \leq s_{1}(1+u)^{\beta} \leq s_{1}$ and

$$
\|S(u) \nabla v(\cdot, t)\|_{L^{q_{1}}(\Omega)} \leq s_{1}\|\nabla v\|_{L^{q_{1}}(\Omega)}<\infty \quad \text { for all } t \in\left(0, T_{\max }\right)
$$

when $\beta>0$, note that $S(u) \leq s_{1}(1+u)^{\beta}$. Using Hölder's inequality yields

$$
\|S(u) \nabla v\|_{L^{q_{1}}(\Omega)} \leq s_{1}\|1+u\|_{L^{p_{0}(\Omega)}}^{\beta} \cdot\|\nabla v\|_{L^{\frac{q_{1} p_{0}}{p_{0}-\beta q_{1}}(\Omega)}}<\infty \quad \text { for all } t \in\left(0, T_{\max }\right) .
$$

Following the same argument, we derive $\|\widetilde{S}(\omega) \nabla z\|_{L^{q_{1}(\Omega)}}<\infty$ for all $t \in\left(0, T_{\max }\right)$. Finally, with the aid of a Moser-type iteration method [38, Lemma A.1], we deduce there exists some positive constant $C_{20}=C_{20}(\beta, \gamma, \widetilde{\gamma})$ such that

$$
\sup _{t \in\left(0, T_{\max }\right)}\|u\|_{L^{\infty}(\Omega)} \leq C_{20} \quad \text { and } \sup _{t \in\left(0, T_{\max }\right)}\|\omega\|_{L^{\infty}(\Omega)} \leq C_{20}
$$

Note that $v(x, t)$ solves

$$
\begin{cases}v_{t}-\Delta v+v=g(\omega), & (x, t) \in \Omega \times\left(0, T_{\max }\right), \\ \frac{\partial v}{\partial \nu}=0, & (x, t) \in \partial \Omega \times\left(0, T_{\max }\right), \\ v(x, 0)=v_{0}(x), & x \in \Omega .\end{cases}
$$

Applying the variation-of-constants formula to $v(x, t)$ and the solution estimates for the heat equation with zero Neumann boundary condition [17, 22, 44 leads to

$$
v(x, t)=e^{t(\triangle-1)} v_{0}+\int_{0}^{t} e^{(t-s)(\triangle-1)} g(\omega(x, s)) .
$$

Meanwhile, choosing $\theta \in\left(\frac{2 n+1}{2(n+1)}, 1-\frac{n(\gamma-1)}{2(n+1)}\right)$, we obtain from 2.1) and 2.2 there exists some positive constant $C$ such that

$$
\begin{aligned}
\|v\|_{W^{1, \infty}(\Omega)} & \leq C\left\|A^{\theta} v\right\|_{L^{n+1}(\Omega)} \\
& \leq C\left\|A^{\theta} e^{t(\triangle-1)} v_{0}\right\|_{L^{n+1}(\Omega)}+C \int_{0}^{t}\left\|A^{\theta} e^{(t-s)(\triangle-1)} g(\omega)\right\|_{L^{n+1}(\Omega)} \\
& \leq C t^{-\theta} e^{-\mu t}\left\|v_{0}\right\|_{L^{n+1}(\Omega)}+C \lambda_{1} \int_{0}^{t}(t-s)^{-\theta-\frac{n}{2} \frac{\gamma-1}{n+1}} e^{-\mu(t-s)}\|\omega\|_{L^{n+1}(\Omega)}^{\gamma} \\
& \leq C\left[t^{-\theta}+\Gamma\left(1-\theta-\frac{n}{2} \frac{\gamma-1}{n+1}\right)\right] \quad \text { for all } t \in\left(0, T_{\max }\right),
\end{aligned}
$$

where $\Gamma(\cdot)$ denotes the Gamma function. Especially, we get

$$
\|v\|_{W^{1, \infty}(\Omega)} \leq C\left[t^{-\theta}+\Gamma\left(1-\theta-\frac{n}{2} \frac{\gamma-1}{n+1}\right)\right] \leq C\left[t_{0}^{-\theta}+\Gamma\left(1-\theta-\frac{n}{2} \frac{\gamma-1}{n+1}\right)\right]
$$

for all $t \in\left(t_{0}, T_{\max }\right)$. This leads to $\sup _{t \in\left(t_{0}, T_{\max }\right)}\|v\|_{L^{\infty}(\Omega)} \leq C_{21}$. It is obvious that $\sup _{t \in\left[0, t_{0}\right]}\|v\|_{L^{\infty}(\Omega)}<\infty$ by Proposition 2.1. Therefore, we get $\sup _{t \in\left(0, T_{\max }\right)}\|v\|_{L^{\infty}(\Omega)}<$ 
$\infty$. Similarly, applying the variation-of-constants formula to $z(x, t)$, following the same argument as $v(x, t)$, we can also infer $\sup _{t \in\left(t_{0}, T_{\max }\right)}\|z\|_{W^{1, \infty}(\Omega)} \leq \widetilde{C}_{21}$. In particular, we get $\sup _{t \in\left(0, T_{\max }\right)}\|z\|_{L^{\infty}(\Omega)} \leq \widetilde{C}_{21}$. As summarized above, we get

$$
\sup _{t \in\left(0, T_{\max }\right)}\left(\|u\|_{L^{\infty}(\Omega)}+\|v\|_{L^{\infty}(\Omega)}+\|\omega\|_{L^{\infty}(\Omega)}+\|z\|_{L^{\infty}(\Omega)}\right)<\infty .
$$

By Proposition 2.1, we have $T_{\max }=\infty$. This completes the proof.

\subsection{Boundedness with logistic source}

In this subsection, we first employ the variation-of-constants formula and maximal Sobolev regularity to establish the following useful proposition.

Proposition 3.2. Let $n \geq 2$ and nonnegative initial data $\left(u_{0}, v_{0}, \omega_{0}, z_{0}\right)$ satisfy (1.5). Suppose $D, S, \widetilde{D}, \widetilde{S}$ and $f, \widetilde{f}, g, \widetilde{g}$ fulfill (1.6) and (1.7) respectively. If $k=\widetilde{k}, \beta=\widetilde{\beta}$ and one of the following conditions holds

(1) $\max \{\gamma, \widetilde{\gamma}\}<k-\beta$;

(2) $\widetilde{\gamma}<\gamma=k-\beta, \mu>2^{k+1} s_{1}, \widetilde{\mu}>\widetilde{c}_{p}$

(3) $\gamma<\widetilde{\gamma}=k-\beta, \mu>c_{p}, \widetilde{\mu}>2^{k+1} \widetilde{s}_{1}$;

(4) $\gamma=\widetilde{\gamma}=k-\beta, \mu>\max \left\{2^{k+1} s_{1}, c_{p}\right\}, \widetilde{\mu}>\max \left\{2^{k+1} \widetilde{s}_{1}, \widetilde{c}_{p}\right\}$,

then for any $p \in[1, \infty)$, there exists some positive constant $C=C(p, \beta, \gamma, \mu, k, \widetilde{\gamma}, \widetilde{\mu})$ such that

$$
\|u(\cdot, t)\|_{L^{p}(\Omega)} \leq C,\|\omega(\cdot, t)\|_{L^{p}(\Omega)} \leq C \quad \text { for all } t \in\left(0, T_{\max }\right) .
$$

Here $c_{p}$ and $\widetilde{c}_{p}$ are defined in (3.41) and 3.39) respectively.

Proof. We only need to prove that for $p$ satisfying $p>\max \{1,2-\beta\}$ there exists some positive constant $C$ such that

$$
\|u(\cdot, t)\|_{L^{p}(\Omega)} \leq C, \quad\|\omega(\cdot, t)\|_{L^{p}(\Omega)} \leq C \quad \text { for all } t \in\left(0, T_{\max }\right) .
$$

Let $t_{0}:=\min \left\{1, \frac{1}{2} T_{\max }\right\}$ and $p>\max \{1,2-\beta\}$ as well as $t \in\left(t_{0}, T_{\max }\right)$. Multiplying the first equation in $(1.4)$ by $(1+u)^{p-1}$, then 1.6 and 1.7$)$ lead to that there exists some positive constant $C_{1}=C_{1}(p, r, \mu)$ such that

$$
\begin{aligned}
\frac{1}{p} \frac{d}{d t} \int_{\Omega}(1+u)^{p}= & -(p-1) \int_{\Omega}(1+u)^{p-2} D(u)|\nabla u|^{2} \\
& +(p-1) \int_{\Omega}(1+u)^{p-2} S(u) \nabla u \cdot \nabla v+\int_{\Omega}(1+u)^{p-1} f(u)
\end{aligned}
$$




$$
\begin{aligned}
\leq & (p-1) \int_{\Omega}(1+u)^{p-2} S(u) \nabla u \cdot \nabla v+r \int_{\Omega}(1+u)^{p-1} u \\
& -\mu \int_{\Omega}(1+u)^{p-1} u^{k} \\
\leq & (p-1) \int_{\Omega}(1+u)^{p-2} S(u) \nabla u \cdot \nabla v+r \int_{\Omega}(1+u)^{p-1} u \\
& -\frac{\mu}{2^{k-1}} \int_{\Omega}(1+u)^{p+k-1}+\mu \int_{\Omega}(1+u)^{p-1} \\
\leq & (p-1) \int_{\Omega}(1+u)^{p-2} S(u) \nabla u \cdot \nabla v+2 r_{+} \int_{\Omega}(1+u)^{p} \\
& -\frac{\mu}{2^{k-1}} \int_{\Omega}(1+u)^{p+k-1}+C_{1} .
\end{aligned}
$$

Let $m=\frac{p+k-1}{k-\beta}$, based on 3.32 as well as Young's inequality, we get there exists some positive constant $C_{2}=C_{2}(p, r, \mu, k)$ such that

$$
\begin{aligned}
& \frac{1}{p} \frac{d}{d t} \int_{\Omega}(1+u)^{p}+\frac{m}{2 p} \int_{\Omega}(1+u)^{p} \\
\leq & (p-1) \int_{\Omega}(1+u)^{p-2} S(u) \nabla u \cdot \nabla v+\left(\frac{m}{2 p}+2 r_{+}\right) \int_{\Omega}(1+u)^{p} \\
& -\frac{\mu}{2^{k-1}} \int_{\Omega}(1+u)^{p+k-1}+C_{1} \\
\leq & (p-1) \int_{\Omega}(1+u)^{p-2} S(u) \nabla u \cdot \nabla v-\frac{\mu}{2^{k}} \int_{\Omega}(1+u)^{p+k-1}+C_{2} .
\end{aligned}
$$

Let

$$
\phi_{1}(\zeta)=(p-1) \int_{0}^{\zeta}(1+\sigma)^{p-2} S(\sigma) \quad \text { for } \zeta \geq 0 .
$$

Thanks to the assumption (1.6), we derive

$$
0 \leq \phi_{1}(\zeta) \leq \frac{s_{1}(p-1)}{p+\beta-1}(1+\zeta)^{p+\beta-1}, \quad \zeta \geq 0 .
$$

Due to $k>\beta$, we see $p+\beta-1=(m-1)(k-\beta)>0$ implies $m>1$. Integrating by parts over $\Omega$, we therefore infer that there exists some positive constant $C_{3}$ such that

$$
\begin{aligned}
& (p-1) \int_{\Omega}(1+u)^{p-2} S(u) \nabla u \cdot \nabla v \\
= & \int_{\Omega} \nabla \phi_{1}(u) \cdot \nabla v \leq \frac{s_{1}(p-1)}{p+\beta-1} \int_{\Omega}(1+u)^{p+\beta-1}|\triangle v| \\
\leq & \frac{\mu}{2^{k+1}} \int_{\Omega}(1+u)^{p+k-1}+C_{3} \int_{\Omega}|\triangle v|^{m},
\end{aligned}
$$

where $C_{3}=C_{3}(p, \beta, \mu, k)=\frac{s_{1}(p-1)(k-\beta)}{(p+\beta-1)(p+k-1)}\left[\frac{2^{k+1} s_{1}}{\mu} \cdot \frac{p-1}{p+k-1}\right]^{\frac{p+\beta-1}{k-\beta}}$. Inserting (3.34) into 3.33 produces

$$
\frac{1}{p} \frac{d}{d t} \int_{\Omega}(1+u)^{p}+\frac{m}{2 p} \int_{\Omega}(1+u)^{p} \leq-\frac{\mu}{2^{k+1}} \int_{\Omega}(1+u)^{p+k-1}+C_{3} \int_{\Omega}|\triangle v|^{m}+C_{2} .
$$


Along with the variation-of-constants formula, we deduce there exists some positive constant $C_{4}$ such that

$$
\begin{aligned}
\frac{1}{p} \int_{\Omega}(1+u)^{p} \leq & -\frac{\mu}{2^{k+1}} \int_{t_{0}}^{t} \int_{\Omega} e^{-\frac{m}{2}(t-s)}(1+u)^{p+k-1}+C_{3} \int_{t_{0}}^{t} \int_{\Omega} e^{-\frac{m}{2}(t-s)}|\triangle v|^{m} \\
& +C_{2} \int_{t_{0}}^{t} e^{-\frac{m}{2}(t-s)}+\frac{1}{p} e^{-\frac{m}{2}\left(t-t_{0}\right)} \int_{\Omega}\left(1+u\left(t_{0}\right)\right)^{p} \\
\leq & -\frac{\mu}{2^{k+1}} \int_{t_{0}}^{t} \int_{\Omega} e^{-\frac{m}{2}(t-s)}(1+u)^{p+k-1}+C_{3} e^{-\frac{m}{2} t} \int_{t_{0}}^{t} \int_{\Omega} e^{\frac{m}{2} s}|\Delta v|^{m}+C_{4}
\end{aligned}
$$

where $C_{4}=: \frac{2 C_{2}}{m}+\frac{1}{p} \int_{\Omega}\left(1+u\left(t_{0}\right)\right)^{p}=C_{4}(p, r, \mu, k, \beta)$. In view of Lemma 2.6 and 3.35), we get there exist positive constants $C_{m}$ and $C_{5}$ such that

$$
\begin{aligned}
\frac{1}{p} \int_{\Omega}(1+u)^{p} \leq & -\frac{\mu}{2^{k+1}} \int_{t_{0}}^{t} \int_{\Omega} e^{-\frac{m}{2}(t-s)}(1+u)^{p+k-1} \\
& +C_{3} C_{m} \int_{t_{0}}^{t} \int_{\Omega} e^{-\frac{m}{2}(t-s)} \lambda_{1}^{m} \omega^{m \gamma}(s) \\
& +C_{3} C_{m} e^{-\frac{m}{2}\left(t-t_{0}\right)}\left[\left\|v\left(\cdot, t_{0}\right)\right\|_{L^{m}(\Omega)}^{m}+\left\|\triangle v\left(\cdot, t_{0}\right)\right\|_{L^{m}(\Omega)}^{m}\right]+C_{4} \\
\leq & -\frac{\mu}{2^{k+1}} \int_{t_{0}}^{t} \int_{\Omega} e^{-\frac{m}{2}(t-s)}(1+u)^{p+k-1} \\
& +C_{3} C_{m} \lambda_{1}^{m} \int_{t_{0}}^{t} \int_{\Omega} e^{-\frac{m}{2}(t-s)} \omega^{m \gamma}(s)+C_{5}
\end{aligned}
$$

where $C_{5}=C_{5}(p, \gamma, \mu, k, \beta)$. Similarly, from the third and fourth equations of (1.4), we deduce there exist positive constants $\widetilde{C}_{3}, \widetilde{C}_{m}$ and $\widetilde{C}_{5}$ such that

$$
\begin{aligned}
\frac{1}{p} \int_{\Omega}(1+\omega)^{p} \leq & -\frac{\widetilde{\mu}}{2^{k+1}} \int_{t_{0}}^{t} \int_{\Omega} e^{-\frac{m}{2}(t-s)}(1+\omega)^{p+k-1} \\
& +\widetilde{C}_{3} \widetilde{C}_{m} \widetilde{\lambda}_{1}^{m} \int_{t_{0}}^{t} \int_{\Omega} e^{-\frac{m}{2}(t-s)} u^{m \widetilde{\gamma}}(s)+\widetilde{C}_{5}
\end{aligned}
$$

where $\widetilde{C}_{5}=\widetilde{C}_{5}(p, \widetilde{\gamma}, \widetilde{\mu}, k, \beta)$. Summing up 3.36 and 3.37p leads to

$$
\begin{aligned}
& \frac{1}{p} \int_{\Omega}(1+u)^{p}+\frac{1}{p} \int_{\Omega}(1+\omega)^{p} \\
\leq & -\frac{\mu}{2^{k+1}} \int_{t_{0}}^{t} \int_{\Omega} e^{-\frac{m}{2}(t-s)}(1+u)^{p+k-1}+\widetilde{C}_{3} \widetilde{C}_{m} \widetilde{\lambda}_{1}^{m} \int_{t_{0}}^{t} \int_{\Omega} e^{-\frac{m}{2}(t-s)} u^{m \widetilde{\gamma}}(s)+\widetilde{C}_{5} \\
& -\frac{\widetilde{\mu}}{2^{k+1}} \int_{t_{0}}^{t} \int_{\Omega} e^{-\frac{m}{2}(t-s)}(1+\omega)^{p+k-1}+C_{3} C_{m} \lambda_{1}^{m} \int_{t_{0}}^{t} \int_{\Omega} e^{-\frac{m}{2}(t-s)} \omega^{m \gamma}(s)+C_{5} .
\end{aligned}
$$

To obtain the boundedness of $\int_{\Omega}(1+u)^{p}$ and $\int_{\Omega}(1+\omega)^{p}$, we subdivide the relationship between $m \gamma$ and $p+k-1$ as well as $m \tilde{\gamma}$ and $p+k-1$ into four cases as below. 
Case 1. When $m \widetilde{\gamma}<p+k-1$ and $m \gamma<p+k-1$, applying Young's inequality to the integrals $\int_{t_{0}}^{t} \int_{\Omega} e^{-\frac{m}{2}(t-s)} u^{m \widetilde{\gamma}}(s)$ and $\int_{t_{0}}^{t} \int_{\Omega} e^{-\frac{m}{2}(t-s)} \omega^{m \gamma}(s)$, we derive there exists some positive constant $C_{6}$ such that

$$
\frac{1}{p} \int_{\Omega}(1+u)^{p}+\frac{1}{p} \int_{\Omega}(1+\omega)^{p} \leq C_{6}
$$

Case 2. When $m \widetilde{\gamma}<p+k-1$ and $m \gamma=p+k-1$, applying Young's inequality to the integral $\int_{t_{0}}^{t} \int_{\Omega} e^{-\frac{m}{2}(t-s)} u^{m \widetilde{\gamma}}(s)$, we can see there exists some positive constant $C_{7}$ such that

$$
\frac{1}{p} \int_{\Omega}(1+u)^{p}+\frac{1}{p} \int_{\Omega}(1+\omega)^{p} \leq-\left[\frac{\widetilde{\mu}}{2^{k+1}}-C_{3} C_{m} \lambda_{1}^{m}\right] \int_{t_{0}}^{t} \int_{\Omega} e^{-\frac{m}{2}(t-s)}(1+\omega)^{p+k-1}+C_{7} .
$$

Thanks to the assumption $\mu>2^{k+1} s_{1}$, we deduce

$$
C_{3} C_{m} \lambda_{1}^{m} 2^{k+1}
$$

$$
\begin{aligned}
& =2^{k+1} C_{m} \lambda_{1}^{m} s_{1}(k-\beta) \frac{p-1}{(p+\beta-1)(p+k-1)}\left(\frac{2^{k+1} s_{1}}{\mu}\right)^{\frac{p+\beta-1}{k-\beta}}\left(\frac{p-1}{p+k-1}\right)^{\frac{p+\beta-1}{k-\beta}} \\
& \leq 2^{k+1} C_{m} \lambda_{1}^{m} s_{1}(k-\beta):=\widetilde{c}_{p}=\widetilde{c}_{p}(k, \beta, p) .
\end{aligned}
$$

By virtue of $\widetilde{\mu}>\widetilde{c}_{p}$, collecting (3.38) and (3.39) results in there exists some positive constant $C_{8}$ such that

$$
\frac{1}{p} \int_{\Omega}(1+u)^{p}+\frac{1}{p} \int_{\Omega}(1+\omega)^{p} \leq C_{8}
$$

Case 3. When $m \widetilde{\gamma}=p+k-1$ and $m \gamma<p+k-1$. By the same procedure to Case 2, we deduce there exists some positive constant $C_{9}$ such that

$$
\frac{1}{p} \int_{\Omega}(1+u)^{p}+\frac{1}{p} \int_{\Omega}(1+\omega)^{p} \leq-\left[\frac{\mu}{2^{k+1}}-\widetilde{C}_{3} \widetilde{C}_{m} \widetilde{\lambda}_{1}^{m}\right] \int_{t_{0}}^{t} \int_{\Omega} e^{-\frac{m}{2}(t-s)}(1+u)^{p+k-1}+C_{9} .
$$

Thanks to the assumption $\widetilde{\mu}>2^{k+1} \widetilde{s}_{1}$, we have

$$
\widetilde{C}_{3} \widetilde{C}_{m} \widetilde{\lambda}_{1}^{m} 2^{k+1}
$$

$$
\begin{aligned}
& =2^{k+1} \widetilde{C}_{m} \widetilde{\lambda}_{1}^{m} \widetilde{s}_{1}(k-\beta) \frac{p-1}{(p+\beta-1)(p+k-1)}\left(\frac{2^{k+1} \widetilde{s}_{1}}{\widetilde{\mu}}\right)^{\frac{p+\beta-1}{k-\beta}}\left(\frac{p-1}{p+k-1}\right)^{\frac{p+\beta-1}{k-\beta}} \\
& \leq 2^{k+1} \widetilde{C}_{m} \widetilde{\lambda}_{1}^{m} \widetilde{s}_{1}(k-\beta):=c_{p}=c_{p}(k, \beta, p) .
\end{aligned}
$$

In view of $\mu>c_{p}$, combining (3.40) and (3.41) shows that there exists some positive constant $C_{10}$ such that

$$
\frac{1}{p} \int_{\Omega}(1+u)^{p}+\frac{1}{p} \int_{\Omega}(1+\omega)^{p} \leq C_{10}
$$


Case 4. When $m \tilde{\gamma}=p+k-1$ and $m \gamma=p+k-1$. Following the same argument as in Cases 2 and 3, we obtain there exists some positive constant $C_{11}$ such that

$$
\begin{aligned}
\frac{1}{p} \int_{\Omega}(1+u)^{p}+\frac{1}{p} \int_{\Omega}(1+\omega)^{p} & \leq-\left[\frac{\mu}{2^{k+1}}-\widetilde{C}_{3} \widetilde{C}_{m} \widetilde{\lambda}_{1}^{m}\right] \int_{t_{0}}^{t} \int_{\Omega} e^{-\frac{m}{2}(t-s)}(1+u)^{p+k-1} \\
& \leq-\left[\frac{\widetilde{\mu}}{2^{k+1}}-C_{3} C_{m} \lambda_{1}^{m}\right] \int_{t_{0}}^{t} \int_{\Omega} e^{-\frac{m}{2}(t-s)}(1+\omega)^{p+k-1}+C_{11} .
\end{aligned}
$$

With the assumptions $\mu>\max \left\{2^{k+1} s_{1}, c_{p}\right\}$ and $\widetilde{\mu}>\max \left\{2^{k+1} \widetilde{s}_{1}, \widetilde{c}_{p}\right\}$ at hand, we derive there exists some positive constant $C_{12}$ such that

$$
\frac{1}{p} \int_{\Omega}(1+u)^{p}+\frac{1}{p} \int_{\Omega}(1+\omega)^{p} \leq C_{12}
$$

As described above, we arrive at the desired result.

With Proposition 3.2 at hand, we can prove Theorem 1.2 as follows.

Proof of Theorem 1.2. We first take $p_{0}>\max \{1,2-\beta\}$ large enough fulfilling (A.8)(A.10) in 38. With Proposition 3.2 at hand, we can find some constant $C_{13}=C_{13}(\beta, \gamma, r$, $\mu, k, \widetilde{\gamma}, \widetilde{r}, \widetilde{\mu})>0$ satisfying

$$
\sup _{t \in\left(0, T_{\max }\right)}\|u\|_{L^{p_{0}(\Omega)}} \leq C_{13} \quad \text { and } \quad \sup _{t \in\left(0, T_{\max }\right)}\|\omega\|_{L^{p_{0}(\Omega)}} \leq C_{13}
$$

Next, choosing $q_{1}>n+2$ and $q_{2}>\frac{n+2}{2}$ such that

$$
\begin{aligned}
(S(u) \nabla v, \widetilde{S}(\omega) \nabla z) & \in\left(L^{\infty}\left(\left(t_{0}, T_{\max }\right), L^{q_{1}}(\Omega)\right)\right)^{2}, \\
(f(u), \widetilde{f}(\omega)) & \in\left(L^{\infty}\left(\left(t_{0}, T_{\max }\right), L^{q_{2}}(\Omega)\right)\right)^{2} .
\end{aligned}
$$

In fact, in one case, note that $\sup _{t \in\left(0, T_{\max }\right)}\|\omega\|_{L^{n+1}(\Omega)}<\infty$, we apply the variation-ofconstants formula once more to $v(x, t)$ as the proof of Theorem 1.1 to get

$$
\sup _{t \in\left(t_{0}, T_{\max }\right)}\|v\|_{W^{1, \infty}(\Omega)}<\infty \quad \text { and } \sup _{t \in\left(t_{0}, T_{\max }\right)}\|z\|_{W^{1, \infty}(\Omega)}<\infty .
$$

And then when $\beta \leq 0$, we infer

$$
\|S(u) \nabla v\|_{L^{q_{1}}(\Omega)} \leq s_{1}\|\nabla v\|_{L^{\infty}(\Omega)}|\Omega|^{\frac{1}{q_{1}}}<\infty \quad \text { for all } t \in\left(t_{0}, T_{\max }\right) ;
$$

meanwhile when $\beta>0$, we can see

$$
\|S(u) \nabla v\|_{L^{q_{1}(\Omega)}} \leq s_{1}\|\nabla v\|_{L^{\infty}(\Omega)}\|1+u\|_{L^{\beta q_{1}(\Omega)}}^{\beta}<\infty \quad \text { for all } t \in\left(t_{0}, T_{\max }\right)
$$

In summary, we deduce $S(u) \nabla v \in L^{q_{1}}(\Omega)$ for all $t \in\left(t_{0}, T_{\max }\right)$. Similarly, we obtain $\widetilde{S}(\omega) \nabla z \in L^{q_{1}}(\Omega)$ for all $t \in\left(t_{0}, T_{\max }\right)$. In another case, note that $f(u) \leq r u-\mu u^{k}$, we 
easily get $\|f(u)\|_{L^{q_{2}(\Omega)}} \leq r\|u\|_{L^{q_{2}(\Omega)}}+\mu\|u\|_{L^{k q_{2}(\Omega)}}^{k}<\infty$ for all $t \in\left(0, T_{\max }\right)$. Similarly we have $\|\tilde{f}(\omega)\|_{L^{q_{2}(\Omega)}}<\infty$ for all $t \in\left(0, T_{\max }\right)$. Finally, with 3.42 at hand, with the aid of a Moser-type iteration method [38, Lemma A.1], we get

$$
\sup _{t \in\left(t_{0}, T_{\max }\right)}\|u\|_{L^{\infty}(\Omega)} \leq C_{14}, \quad \sup _{t \in\left(t_{0}, T_{\max }\right)}\|\omega\|_{L^{\infty}(\Omega)} \leq C_{14}
$$

where $C_{14}=C_{14}(\beta, \gamma, \widetilde{\gamma}, r, \widetilde{r}, \mu, \widetilde{\mu}, k)$. It is obvious that $\sup _{t \in\left[0, t_{0}\right]}\|u\|_{L^{\infty}(\Omega)}<\infty$ and $\sup _{t \in\left[0, t_{0}\right]}\|\omega\|_{L^{\infty}(\Omega)}<\infty$ by Proposition 2.1. In summary, with the aid of the above estimates, we infer

$$
\sup _{t \in\left(0, T_{\max }\right)}\|u\|_{L^{\infty}(\Omega)}<\infty, \quad \sup _{t \in\left(0, T_{\max }\right)}\|\omega\|_{L^{\infty}(\Omega)}<\infty
$$

As described above, we deduce

$$
\sup _{t \in\left(0, T_{\max }\right)}\left(\|u\|_{L^{\infty}(\Omega)}+\|v\|_{L^{\infty}(\Omega)}+\|\omega\|_{L^{\infty}(\Omega)}+\|z\|_{L^{\infty}(\Omega)}\right)<\infty .
$$

Now $T_{\max }=\infty$ follows from Proposition 2.1 and hence the proof is complete.

\section{Conclusion}

In this article, we studied a fully parabolic quasilinear chemotaxis model with nonlinear signal production. When there is no growth source, nonlinear variants with diffusivity, chemotactic sensitivity and signal production ensure the global existence of the solutions. We also showed that strong logistic damping effect warrants the global existence of the solutions.

It is necessary to point out that under the cases of

$$
f(u)=r u-\mu u^{k}, \quad \widetilde{f}(\omega)=\widetilde{r} \omega-\widetilde{\mu} \omega^{\widetilde{k}}, \quad \widetilde{g}(u)=\widetilde{g}_{1} u^{\widetilde{\gamma}}, \quad g(\omega)=g_{1} \omega^{\gamma},
$$

the stabilities of the solutions remain open. We guess that the bounded solutions warranted by Theorem 1.2 have the property

$$
\begin{aligned}
& \left\|u-\left(\frac{r_{+}}{\mu}\right)^{\frac{1}{k-1}}\right\|_{L^{\infty}(\Omega)} \rightarrow 0, \quad\left\|z-\widetilde{g}_{1}\left(\frac{r_{+}}{\mu}\right)^{\frac{\widetilde{\gamma}}{k-1}}\right\|_{L^{\infty}(\Omega)} \rightarrow 0, \\
& \left\|\omega-\left(\frac{\widetilde{r}_{+}}{\widetilde{\mu}}\right)^{\frac{1}{\tilde{k}-1}}\right\|_{L^{\infty}(\Omega)} \rightarrow 0, \quad\left\|v-g_{1}\left(\frac{\widetilde{r}_{+}}{\widetilde{\mu}}\right)^{\frac{\gamma}{\tilde{k}-1}}\right\|_{L^{\infty}(\Omega)} \rightarrow 0 .
\end{aligned}
$$

If it is true, we can study the convergence rate of the solutions, but unfortunately, we do not make a clear answer to this guess. It is worth mentioning that for single-species chemotaxis systems involving linear diffusion and logistic-type terms, results on large time stabilization 
have already been obtained by Winkler for $k=2$ in [48] and substantially smaller $k$ in [50]. In addition, for a fully parabolic quasilinear single-species chemotaxis model involving general logistic source and signal production, result on large time stabilization has already been achieved by Ding et al. [8]. These may be very helpful to solve our guess. We leave it for future work.

\section{Acknowledgments}

The authors are grateful to the anonymous referees' attentive reading of this manuscript and valuable suggestions which help to significantly improve the paper.

\section{References}

[1] P. Biler, E. E. Espejo and I. Guerra, Blowup in higher dimensional two species chemotactic systems, Commun. Pure Appl. Anal. 12 (2013), no. 1, 89-98.

[2] A. Blanchet, On the parabolic-elliptic Patlak-Keller-Segel system in dimension 2 and higher, in: Séminaire Laurent Schwartz-Équations aux dérivées partielles et applications, Année 2011-2012, Exp. No. VIII, 26 pp., Sémin. Équ. Dériv. Partielles, École Polytech., Palaiseau, 2013.

[3] J. Burczak, T. Cieślak and C. Morales-Rodrigo, Global existence vs. blowup in a fully parabolic quasilinear 1D Keller-Segel system, Nonlinear Anal. 75 (2012), no. 13, $5215-5228$.

[4] V. Calvez and J. A. Carrillo, Volume effects in the Keller-Segel model: Energy estimates preventing blow-up, J. Math. Pures Appl. (9) 86 (2006), no. 2, 155-175.

[5] M. A. J. Chaplain and J. I. Tello, On the stability of homogeneous steady states of a chemotaxis system with logistic growth term, Appl. Math. Lett. 57 (2016), 1-6.

[6] T. Cieślak and P. Laurençot, Finite time blow-up for a one-dimensional quasilinear parabolic-parabolic chemotaxis system, Ann. Inst. H. Poincaré Anal. Non Linéaire 27 (2010), no. 1, 437-446.

[7] T. Cieślak and C. Stinner, Finite-time blowup and global-in-time unbounded solutions to a parabolic-parabolic quasilinear Keller-Segel system in higher dimensions, J. Differential Equations 252 (2012), no. 10, 5832-5851.

[8] M. Ding, W. Wang, S. Zhou and S. Zheng, Asymptotic stability in a fully parabolic quasilinear chemotaxis model with general logistic source and signal production, J. Differential Equations 268 (2020), no. 11, 6729-6777. 
[9] A. Friedman, Partial Differential Equations, Holt, Rinehart and Winston, New York, 1969.

[10] E. Galakhov, O. Salieva and J. I. Tello, On a parabolic-elliptic system with chemotaxis and logistic type growth, J. Differential Equations 261 (2016), no. 8, 4631-4647.

[11] D. D. Haroske and H. Triebel, Distributions, Sobolev Spaces, Elliptic Equations, EMS Textbooks in Mathematics, European Mathematical Society (EMS), Zürich, 2008.

[12] X. He, M. Tian and S. Zheng, Large time behavior of solutions to a quasilinear attraction-repulsion chemotaxis system with logistic source, Nonlinear Anal. Real World Appl. 54 (2020), 103095, 14 pp.

[13] M. E. Hibbing, C. Fuqua, M. R. Parsek and S. B. Peterson, Bacterial competition: surviving and thriving in the microbial jungle, Nat. Rev. Microbiol. 8 (2010), no. 1, $15-25$.

[14] T. Hillen and K. J. Painter, A user's guide to PDE models for chemotaxis, J. Math. Biol. 58 (2009), no. 1-2, 183-217.

[15] T. Hillen and A. Potapov, The one-dimensional chemotaxis model: global existence and asymptotic profile, Math. Methods Appl. Sci. 27 (2004), no. 15, 1783-1801.

[16] D. Horstmann, From 1970 until present: the Keller-Segel model in chemotaxis and its consequences I, Jahresber. Deutsch. Math.-Verein. 105 (2003), no. 3, 103-165.

[17] D. Horstmann and M. Winkler, Boundedness vs. blow-up in a chemotaxis system, J. Differential Equations 215 (2005), no. 1, 52-107.

[18] B. Hu and Y. Tao, Boundedness in a parabolic-elliptic chemotaxis-growth system under a critical parameter condition, Appl. Math. Lett. 64 (2017), 1-7.

[19] S. Ishida, K. Seki and T. Yokota, Boundedness in quasilinear Keller-Segel systems of parabolic-parabolic type on non-convex bounded domains, J. Differential Equations 256 (2014), no. 8, 2993-3010.

[20] E. F. Keller and L. A. Segel, Initiation of slime mold aggregation viewed as an instability, J. Theoret. Biol. 26 (1970), no. 3, 399-415.

[21] _ Model for chemotaxis, J. Theoret. Biol. 30 (1971), no. 2, 225-234.

[22] R. Kowalczyk and Z. Szymańska, On the global existence of solutions to an aggregation model, J. Math. Anal. Appl. 343 (2008), no. 1, 379-398. 
[23] X. Li and Y. Wang, Boundedness in a two-species chemotaxis parabolic system with two chemicals, Discrete Contin. Dyn. Syst. Ser. B 22 (2017), no. 7, 2717-2729.

[24] X. Li and Z. Xiang, Boundedness in quasilinear Keller-Segel equations with nonlinear sensitivity and logistic source, Discrete Contin. Dyn. Syst. 35 (2015), no. 8, 35033531.

[25] D.-m. Liu and Y.-s. Tao, Boundedness in a chemotaxis system with nonlinear signal production, Appl. Math. J. Chinese Univ. Ser. B 31 (2016), no. 4, 379-388.

[26] Y. Liu and Y. Tao, Asymptotic behavior in a chemotaxis-growth system with nonlinear production of signals, Discrete Contin. Dyn. Syst. Ser. B 22 (2017), no. 2, 465-475.

[27] N. Mizoguchi and P. Souplet, Nondegeneracy of blow-up points for the parabolic Keller-Segel system, Ann. Inst. H. Poincaré Anal. Non Linéaire 31 (2014), no. 4, 851-875.

[28] J. D. Murray, Mathematical Biology, Second edition, Biomathematics 19, SpringerVerlag, Berlin, 1993.

[29] M. Negreanu and J. I. Tello, On a two species chemotaxis model with slow chemical diffusion, SIAM J. Math. Anal. 46 (2014), no. 6, 3761-3781.

[30] K. Osaki, T. Tsujikawa, A. Yagi and M. Mimura, Exponential attractor for a chemotaxis-growth system of equations, Nonlinear Anal. 51 (2002), no. 1, Ser. A: Theory Methods, 119-144.

[31] K. Osaki and A. Yagi, Finite dimensional attractor for one-dimensional Keller-Segel equations, Funkcial. Ekvac. 44 (2001), no. 3, 441-469.

[32] K. J. Painter, Continuous models for cell migration in tissues and applications to cell sorting via differential chemotaxis, Bull. Math. Biol. 71 (2009), no. 5, 1117-1147.

[33] K. J. Painter and T. Hillen, Volume-filling and quorum-sensing in models for chemosensitive movement, Can. Appl. Math. Q. 10 (2002), no. 4, 501-543.

[34] K. J. Painter and J. A. Sherratt, Modelling the movement of interacting cell populations, J. Theoret. Biol. 225 (2003), no. 3, 327-339.

[35] H. Qiu and S. Guo, Global existence and stability in a two-species chemotaxis system, Discrete Contin. Dyn. Syst. Ser. B 24 (2019), no. 4, 1569-1587.

[36] C. Stinner, J. I. Tello and M. Winkler, Competitive exclusion in a two-species chemotaxis model, J. Math. Biol. 68 (2014), no. 7, 1607-1626. 
[37] X. Tao, S. Zhou and M. Ding, Boundedness of solutions to a quasilinear parabolicparabolic chemotaxis model with nonlinear signal production, J. Math. Anal. Appl. 474 (2019), no. 1, 733-747.

[38] Y. Tao and M. Winkler, Boundedness in a quasilinear parabolic-parabolic KellerSegel system with subcritical sensitivity, J. Differential Equations 252 (2012), no. 1, $692-715$.

[39] _ Boundedness vs. blow-up in a two-species chemotaxis system with two chemicals, Discrete Contin. Dyn. Syst. Ser. B 20 (2015), no. 9, 3165-3183.

[40] J. I. Tello and M. Winkler, Stabilization in a two-species chemotaxis system with a logistic source, Nonlinearity 25 (2012), no. 5, 1413-1425.

[41] X. Tu, C. Mu, P. Zheng and K. Lin, Global dynamics in a two-species chemotaxiscompetition system with two signals, Discrete Contin. Dyn. Syst. 38 (2018), no. 7, 3617-3636.

[42] L. Wang and C. Mu, A new result for boundedness and stabilization in a two-species chemotaxis system with two chemicals, Discrete Contin. Dyn. Syst. Ser. B 25 (2020), no. $12,4585-4601$.

[43] L. Wang, J. Zhang, C. Mu and X. Hu, Boundedness and stabilization in a two-species chemotaxis system with two chemicals, Discrete Contin. Dyn. Syst. Ser. B 25 (2020), no. 1, 191-221.

[44] M. Winkler, Absence of collapse in a parabolic chemotaxis system with signaldependent sensitivity, Math. Nachr. 283 (2010), no. 11, 1664-1673.

[45]__ Boundedness in the higher-dimensional parabolic-parabolic chemotaxis system with logistic source, Comm. Partial Differential Equations 35 (2010), no. 8, 1516-1537.

[46] _ Does a 'volume-filling effect' always prevent chemotactic collapse?, Math. Methods Appl. Sci. 33 (2010), no. 1, 12-24.

[47] _ Finite-time blow-up in the higher-dimensional parabolic-parabolic KellerSegel system, J. Math. Pures Appl. (9) 100 (2013), no. 5, 748-767.

[48] _ Global asymptotic stability of constant equilibria in a fully parabolic chemotaxis system with strong logistic dampening, J. Differential Equations 257 (2014), no. 4, 1056-1077.

[49] _ A critical blow-up exponent in a chemotaxis system with nonlinear signal production, Nonlinearity 31 (2018), no. 5, 2031-2056. 
[50] - The role of superlinear damping in the construction of solutions to driftdiffusion problems with initial data in $L^{1}$, Adv. Nonlinear Anal. 9 (2020), no. 1, $526-566$.

[51] M. Winkler and K. C. Djie, Boundedness and finite-time collapse in a chemotaxis system with volume-filling effect, Nonlinear Anal. 72 (2010), no. 2, 1044-1064.

[52] T. Xiang, On effects of sampling radius for the nonlocal Patlak-Keller-Segel chemotaxis model, Discrete Contin. Dyn. Syst. 34 (2014), no. 11, 4911-4946.

[53] _ Boundedness and global existence in the higher-dimensional parabolicparabolic chemotaxis system with/without growth source, J. Differential Equations 258 (2015), no. 12, 4275-4323.

[54] _ Dynamics in a parabolic-elliptic chemotaxis system with growth source and nonlinear secretion, Commun. Pure Appl. Anal. 18 (2019), no. 1, 255-284.

[55] H. Yu, W. Wang and S. Zheng, Criteria on global boundedness versus finite time blowup to a two-species chemotaxis system with two chemicals, Nonlinearity 31 (2018), no. $2,502-514$.

[56] Q. Zhang, Competitive exclusion for a two-species chemotaxis system with two chemicals, Appl. Math. Lett. 83 (2018), 27-32.

[57] Q. Zhang and Y. Li, Global boundedness of solutions to a two-species chemotaxis system, Z. Angew. Math. Phys. 66 (2015), no. 1, 83-93.

[58] Q. Zhang, X. Liu and X. Yang, Global existence and asymptotic behavior of solutions to a two-species chemotaxis system with two chemicals, J. Math. Phys. 58 (2017), no. $11,111504,9$ pp.

[59] J. Zheng, Boundedness of solutions to a quasilinear parabolic-elliptic Keller-Segel system with logistic source, J. Differential Equations 259 (2015), no. 1, 120-140.

[60] _ Boundedness of solutions to a quasilinear parabolic-parabolic Keller-Segel system with a logistic source, J. Math. Anal. Appl. 431 (2015), no. 2, 867-888.

[61]__ Boundedness in a two-species quasi-linear chemotaxis system with two chemicals, Topol. Methods Nonlinear Anal. 49 (2017), no. 2, 463-480.

[62] P. Zheng, C. Mu, X. Hu and Y. Tian, Boundedness of solutions in a chemotaxis system with nonlinear sensitivity and logistic source, J. Math. Anal. Appl. 424 (2015), no. 1, $509-522$. 
[63] P. Zheng, C. Mu and Y. Mi, Global existence and decay for a chemotaxis-growth system with generalized volume-filling effect and sublinear secretion, NoDEA Nonlinear Differential Equations Appl. 24 (2017), no. 2, Paper No. 13, 18 pp.

[64] _ Global stability in a two-competing-species chemotaxis system with two chemicals, Differential Integral Equations 31 (2018), no. 7-8, 547-558.

Aichao Liu

School of Mathematics and Statistics, HNP-LAMA, Central South University, Changsha, 410083, China

and

School of Mathematics and Statistics, Huanghuai University, Zhumadian, 463000, China E-mail address: aichaoliu@csu.edu.cn

Binxiang Dai

School of Mathematics and Statistics, HNP-LAMA, Central South University, Changsha, 410083, China

E-mail address: bxdai@csu.edu.cn 